ARTICLE

\title{
Common variants in Alzheimer's disease and risk stratification by polygenic risk scores
}

Genetic discoveries of Alzheimer's disease are the drivers of our understanding, and together with polygenetic risk stratification can contribute towards planning of feasible and efficient preventive and curative clinical trials. We first perform a large genetic association study by merging all available case-control datasets and by-proxy study results (discovery $n=409,435$ and validation size $n=58,190$ ). Here, we add six variants associated with Alzheimer's disease risk (near APP, CHRNE, PRKD3/NDUFAF7, PLCG2 and two exonic variants in the SHARPIN gene). Assessment of the polygenic risk score and stratifying by APOE reveal a 4 to 5.5 years difference in median age at onset of Alzheimer's disease patients in APOE \& 4 carriers. Because of this study, the underlying mechanisms of APP can be studied to refine the amyloid cascade and the polygenic risk score provides a tool to select individuals at high risk of Alzheimer's disease. 
T hus far, multiple loci associated with Alzheimer's disease (AD) have been described next to causal mutations in two subunits of $\gamma$-secretases, membrane-embedded aspartyl complexes (PSEN1, PSEN2 genes), and the gene encoding one target protein of these proteases, the amyloid precursor protein gene (APP). The most prominent locus, APOE, was detected almost 30 years ago using linkage techniques ${ }^{1}$. In addition, genome-wide association studies (GWAS) of AD case-control datasets and by-proxy AD case-control studies have identified 30 genomic loci that modify the risk of $\mathrm{AD}^{2-7}$. These signals account for $\sim 31 \%$ of the genetic variance of $\mathrm{AD}$, leaving most of the genetic risk as yet uncharacterized ${ }^{8}$. Further disentangling the genetic constellation of common genetic variations underlying $\mathrm{AD}$ can drive our biological insights of $\mathrm{AD}$ and can point toward novel drug targets.

There are over 50 million people living with dementia and the global cost of dementia is well above 1 trillion US\$ $\$$. This means there is a medical and economical urgency to efficiently test interventions that are under development. Therefore, to increase power and reduce duration of trials, pre-symptomatic patients that are at high genetic risk of disease are increasingly developed ${ }^{10}$. However, only carriers of causal mutations (APP, $P S E N 1$, and PSEN2) and the APOE \&4 allele are considered high risk, while other common and rare genetic variants are ignored ${ }^{11}$. Despite that, the combined effects of all currently known variants in a polygenic risk score (PRS) is associated with the conversion of mild cognitive impairment to $\mathrm{AD}^{12,13}$, the neuropathological hallmarks of $\mathrm{AD}$, age at onset (AAO) of disease ${ }^{14-17}$ and lifetime risk of $\mathrm{AD}^{18}$.

In this work we aim to comprehend and expand the knowledge of the genetic landscape underlying $\mathrm{AD}$ and provide additional evidence that a PRS of variants can be a robust tool to select high risk individuals with an earlier AAO. We first performed a metaGWAS integrating all currently published GWAS case-control data, by-proxy case-control data, and the data from the Genome Research at Fundació ACE (GR@ACE) study ${ }^{19}$. We confirm the observed associations in a large independent replication study. Then, we construct an update of the PRS and test whether the effects of the PRS are influenced by diagnostic certainty, sex and AAO groups. Lastly, we test whether the PRS could be used to identify individuals at the highest odds of having $\mathrm{AD}$ and we compared $\mathrm{AAO}$ of the $\mathrm{AD}$ cases. This study describes the identification of six variants associated with $\mathrm{AD}$ risk and provides an extended PRS tool to select individuals at high risk of AD.

\section{Results}

Meta-GWAS of AD. We combined data from three AD GWASs: the summary statistics calculated from the GR@ACE ${ }^{19}$ casecontrol study (6331 AD cases and 6055 controls), the IGAP 20 case-control study (up to 30,344 AD cases and 52,427 controls) and the UKB AD-by-proxy case-control study ${ }^{21}$ (27,696 cases of maternal AD with 260,980 controls, and 14,338 cases of paternal AD with 245,941 controls, Fig. 1, Supplementary Data 1). Although we observed inflation in the resulting summary statistics $(\lambda$ median $=1.08$; see Supplementary Fig. $1 d)$, it was not driven by an un-modeled population structure (LD score regression intercept $=1.036$ ). The full details of the studies are described in methods. After study-specific variant filtering and quality-control procedures, we performed a fixed effects inversevariance-weighted meta-analysis ${ }^{22}$ on the summary statistics of the three studies. Using this strategy, we identified a genome-wide significant (GWS) association $\left(p<5 \times 10^{-8}\right)$ for 36 independent genetic variants in 35 genomic regions (the APOE region contains signals for $\varepsilon 4$ and $\varepsilon 2$ ). As a sensitivity analysis, we removed the AD-by-proxy study and compared the resulted effect estimates with and without this dataset. We found a high correlation between the effect estimates from the case-control and by-proxy

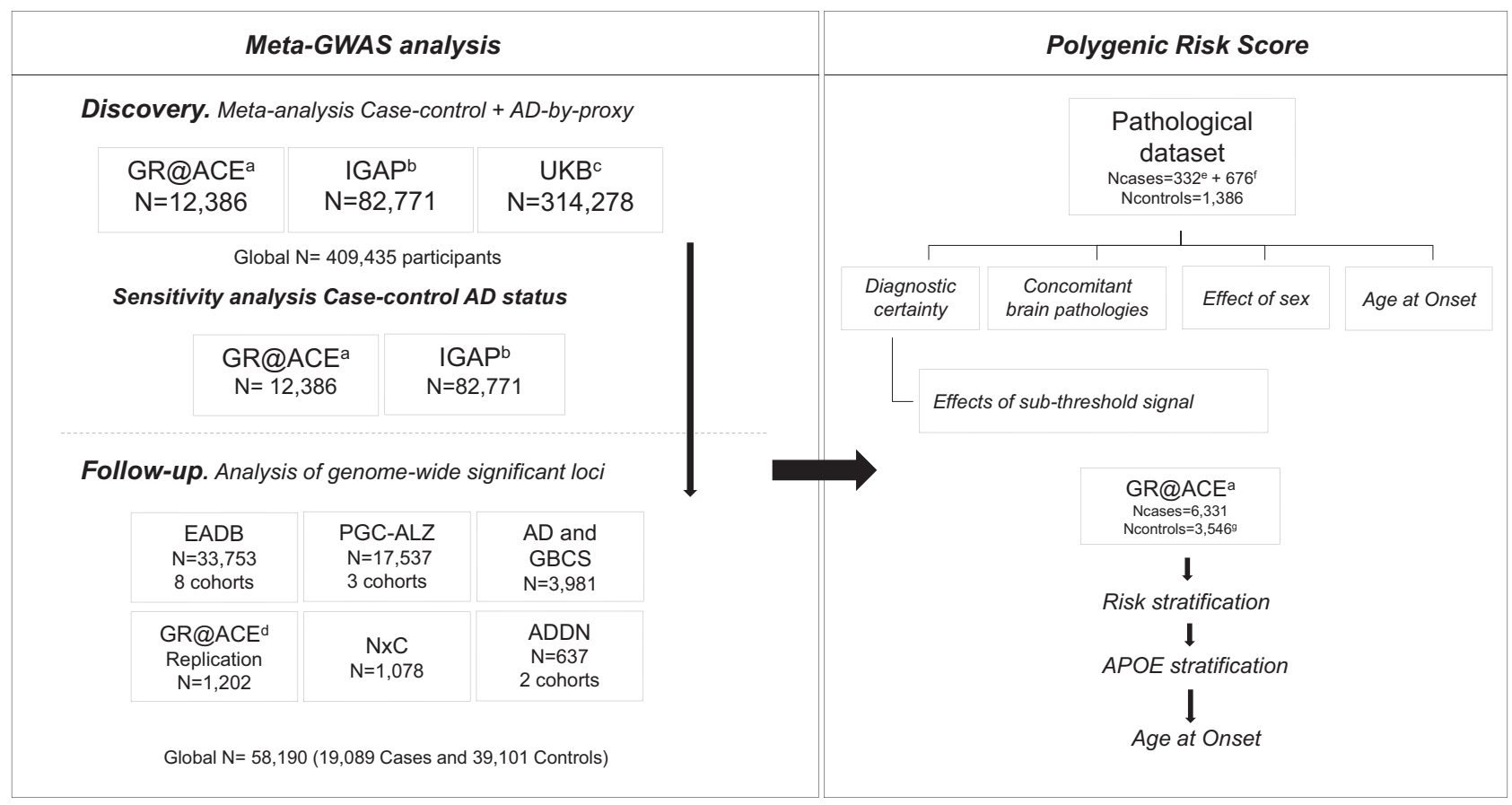

Fig. 1 Flow chart of analysis steps. Discovery meta-analysis in GR@ACE, IGAP stage $1+2$ and UKBiobank followed by a replication in 16 independent cohorts. The genome-wide significant signals found in meta-GWAS were used to perform a Polygenic Risk Score in a clinical and pathological AD dataset.

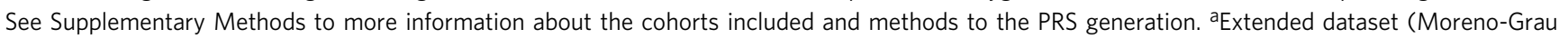
et al. ${ }^{19}$ ), bStagel + Stagell (Kunkle et al. ${ }^{20}$ ), cBy proxy AD: Meta-analysis of maternal and paternal history of dementia (Marioni et al. ${ }^{21}$ ), dExtra and independent GR@ACE dataset incorporated only for replication purposes, ePathologically confirmed AD cases, ${ }^{f} A D$ cases diagnosed based on clinical criteria, ${ }^{8}$ Controls participants aged 55 years and younger. $N=$ Total of individuals within specified data. 
a

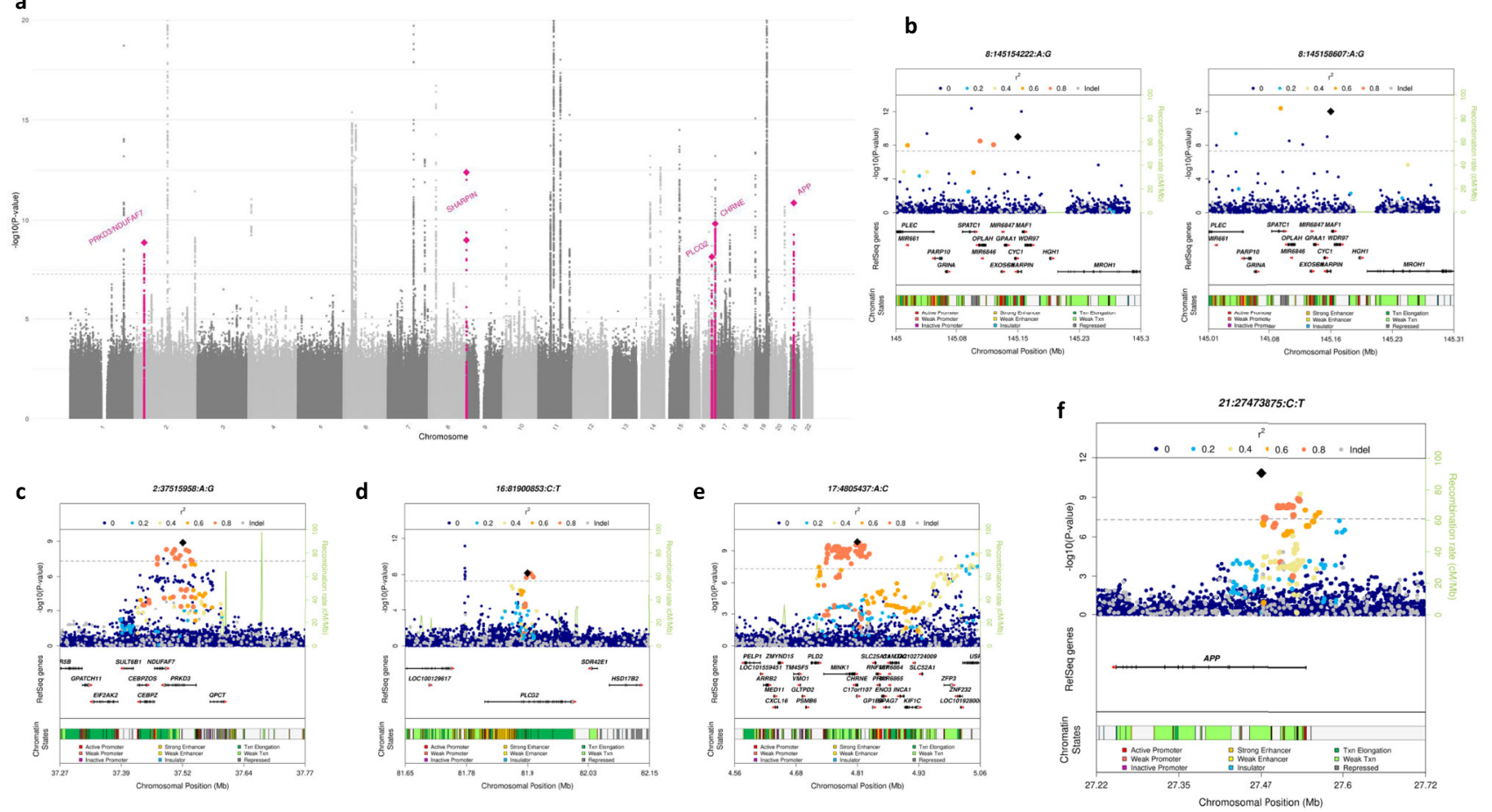

Fig. 2 GWAS meta-analysis for AD risk $(\mathbf{N}=\mathbf{4 6 7 , 6 2 3 ) . ~ a ~ M a n h a t t a n ~ p l o t ~ o f ~ o v e r a l l ~ m e t a - a n a l y s i s ~ f o r ~ g e n o m e - w i d e ~ a s s o c i a t i o n ~ i n ~ A l z h e i m e r ' s ~ d i s e a s e ~}$ highlighting in pink the loci associated with AD in this study (PRKD3/NDUFAF7, SHARPIN, CHRNE, PLCG2, and APP). b-f Locus plots for the signals associated with $A D$ in overall meta-analysis results.

approaches for the significant loci $\left(R^{2}=0.994, p=8.1 \times 10^{-37}\right.$; Supplementary Fig. 1e). Four genomic regions were not previously associated with $\mathrm{AD}$ (see Manhattan Plot, Fig. 2a).

Next, we aimed at replicating the associated loci in 16 cohorts $(19,087 \mathrm{AD}$ cases and 39,101 controls in total), many of them collected and analyzed by the European Alzheimer's Disease Biobank (JPND-EADB) project. We tested all variants with suggestive association $\left(p<10^{-5}\right)$ located within a $200 \mathrm{~kb}$ region from the sentinel SNP. Overall, 384 variants were tested in the replication datasets (Supplementary Data 2). Discovery and replication were combined, and we identified associations in six variants comprising five genomic loci annotated using FUMA ${ }^{23}$ (Table 1, Fig. 2b-f, Supplementary Fig. 2 and Supplementary Results). In $A P P$, we identified a common $(\mathrm{MAF}=0.46)$ intronic variant associated with a reduced risk of $\mathrm{AD}$ ( $\mathrm{rs} 2154481, \mathrm{OR}=0.95$ [0.94-0.96], $p=1.39 \times 10^{-11}$, Fig. 2f). In SHARPIN (SHANK Associated RH Domain Interactor) gene, we found two missense mutations (rs34173062/p.Ser17Phe and rs34674752/p.Pro294Ser) that are in linkage equilibrium $\left(R^{2}=1.3 \times 10^{-6}, D^{\prime}=0.014\right.$, $p=0.96$ ). Both missense variants increased $\mathrm{AD}$ risk (p.Ser17Phe, $\mathrm{MAF}=0.085, \quad \mathrm{OR}=1.14 \quad[1.10-1.18], \quad p=9.6 \times 10^{-13}$ and $\mathrm{p}$. Pro294Ser, $\mathrm{MAF}=0.052, \mathrm{OR}=1.13[1.09-1.18], p=1.0 \times 10^{-9}$, Fig. 2b). A variant close to the genes PRKD3 and NDUFAF7 ( $\mathrm{rs876461}, \mathrm{MAF}=0.143$ ) emerged as the most significant variant in the region after the combined analysis $(\mathrm{OR}=1.07$ [1.05-1.09], $p=1.3 \times 10^{-9}$, Fig. 2c). In the 3'-UTR region of CHRNE (Cholinergic Receptor Nicotinic Epsilon Subunit), rs72835061 $(\mathrm{MAF}=0.085)$ was associated with a 1.09 -fold increased risk of $\mathrm{AD}\left(95 \% \mathrm{CI}[1.06-1.11], p=1.5 \times 10^{-10}\right.$, Fig. 2e). Our analysis also strengthened the evidence of association with $\mathrm{AD}$ for three additional genomic loci including an association with a variant in PLCG2 (rs3935877, MAF $=0.13, \mathrm{OR}=0.92$ [0.90-0.95], $p=6.9 \times$ $10^{-9}$, Fig. 2d), and confirmed another common variant in PLCG2, a stop gain mutation in $I L-34$ and a variant near HS3ST1 (Table 1, Supplementary Fig. 3 and Supplementary Data 2, 3). We were not able to replicate two loci (ELK2AP and SPPL2A regions) that showed suggestive association with $\mathrm{AD}\left(p<1 \times 10^{-7}\right.$ in discovery).

Polygenic risk scores. In order to assess the robustness and combined effect of the genetic landscape of AD (Fig. 3, Supplementary Data 4), we constructed a weighted PRS based on the 39 genetic variants (excluding $A P O E$ genotypes) that showed GWS evidence of association with AD (see Methods, Fig. 4 and Supplementary Data 5). We tested if the association of the PRS with $\mathrm{AD}$ is independent of clinically important factors that are considered in the selection of individuals for clinical trials. First, we showed that the association of the PRS with clinically diagnosed $\mathrm{AD}$ cases is similar to the association with pathologically confirmed $\mathrm{AD}(\mathrm{OR}=1.30$ vs. 1.38 , per $1-\mathrm{SD}$ increase in the PRS). In this setting, adding variants below the GWS threshold did not lead to a more significant association of the PRS with AD (Fig. 4a). Next, we tested whether the PRS was associated with $\mathrm{AD}$ in the presence of concomitant brain pathologies (besides $\mathrm{AD}$ ). Among our autopsy-confirmed $\mathrm{AD}$ patients ( $n=332), 84 \%$ had at least one concomitant pathology, and the PRS was associated with AD in the presence of all tested concomitant pathologies (Fig. 4b). Moreover, the patients often had more than one concomitant pathology (48.8\%), but no difference was observed in the effect estimate of the PRS when more than one pathology was present (Fig. 4b). Last, we investigated the effect of sex and AAO (Fig. 4c). Our analysis revealed that the effect of the PRS was the same in both sexes (Fig. 4c) and was consistent with both early-onset (onset before 65 years; $\left.\mathrm{OR}=1.58,95 \% \mathrm{CI}[1.22-2.05], p=5.8 \times 10^{-4}\right)$ as well as with late-onset $\mathrm{AD}$ (onset later than 85 years; $\mathrm{OR}=1.29,95 \%$ CI [1.10-1.51], $\left.p=1.5 \times 10^{-3}\right)$.

PRSs has the potential to early identify subjects at risk of complex diseases ${ }^{24}$. To identify people at the highest genetic risk of $\mathrm{AD}$ based on the PRS, we used the validated 39-variants 


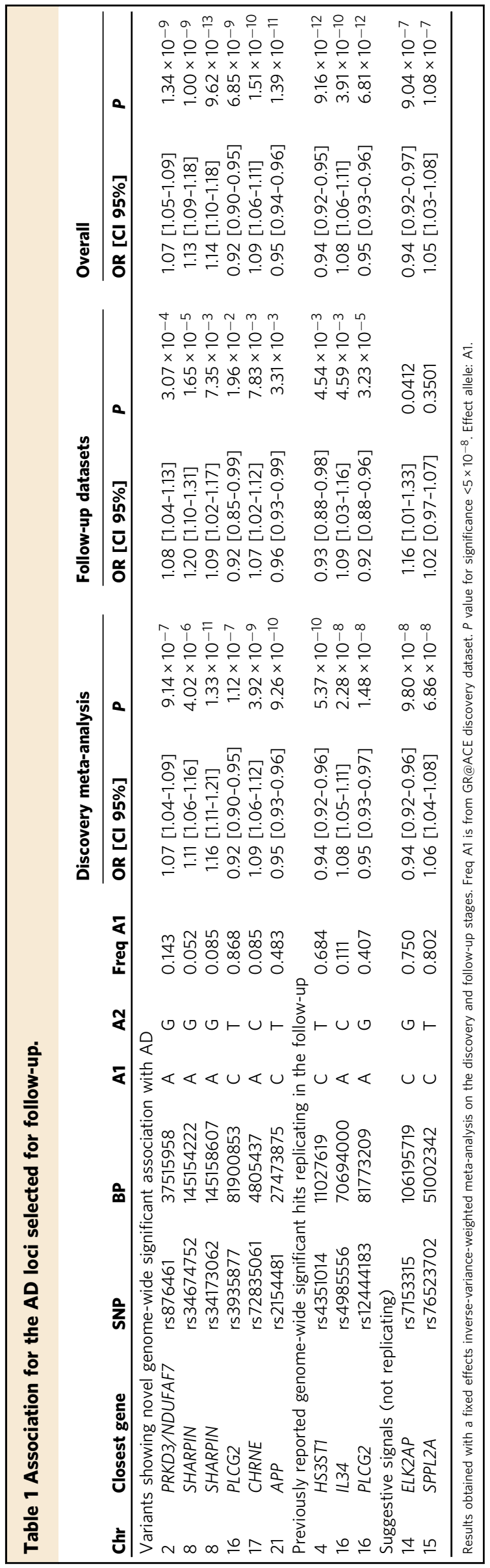

PRS in the large GR@ACE dataset. The PRS was associated with a 1.27-fold (95\% CI [1.23-1.32]) increased risk for every standard deviation increase in the PRS $\left(p=7.3 \times 10^{-39}\right)$ and with a gradual risk increase when we stratified the dataset into $2 \%$ percentiles of the PRS (Fig. 5a, Supplementary Data 6). Next, we stratified the dataset in APOE genotype risk groups. The PRS percentiles were associated with $\mathrm{AD}$ within the APOE genotype groups (Fig. 5b, Supplementary Data 7). Finally, we compared the risk extremes and found a 16.2-fold (95\% CI [8.84-29.5], $\left.p=1.5 \times 10^{-19}\right)$ increased risk for the highest-PRS group ( $A P O E$ $\varepsilon 4 \varepsilon 4)$ compared with the lowest-PRS group (APOE $\varepsilon 2 \varepsilon 2 / \varepsilon 2 \varepsilon 3$; Supplementary Data 8). When we compared the median AAO in $\mathrm{AD}$ patients in these extreme risk groups we found a 9-year difference in the median age $\left(p_{\text {Wilcoxon }}=1.7 \times 10^{-6}\right)$ (Fig. $5 \mathrm{c}$ ). Lastly, we studied the effects on AAO of the PRS in the APOE genotype groups. The PRS differentiated AAO only within APOE $\varepsilon 4$ carriers. In $A P O E \varepsilon 4$ heterozygotes the PRS determined a 4 year difference in median AAO and in APOE $\varepsilon 4$ homozygotes $\left(p_{\text {Wilcoxon }}=6.9 \times 10^{-5}\right)$, where the PRS determined a median AAO difference of 5.5 years $\left(p_{\text {Wilcoxon }}=4.6 \times 10^{-5}\right)$. For the selection of high-risk individuals, it is important to note that we found no difference in the odds and $\mathrm{AAO}$ for $\mathrm{AD}$ for $A P O E \varepsilon 4$ heterozygotes with the highest PRS compared to APOE $\varepsilon 4$ homozygotes with the lowest PRS. The Cox regression also showed an impact of $A P O E$ on AAO, mainly on APOE $\varepsilon 4 \varepsilon 4$ (significant $A P O E-P R S$ interaction $(p=0.021)$, Fig. 5d, Supplementary Data 9).

\section{Discussion}

This work adds on the ongoing global effort to identify genetic variants associated with $\mathrm{AD}$ (Fig. 3). In the present work, we reported on the largest GWAS for AD risk to date, comprising genetic information of 467,623 individuals of European ancestry. We identified six variants that were not previously associated with the risk of $\mathrm{AD}$ and constructed a robust $\mathrm{PRS}$ for $\mathrm{AD}$ demonstrating its potential value for selecting subjects at risk of $\mathrm{AD}$, especially within APOE \&4 carriers. This PRS was based on European ancestries and may or may not generalize to other ancestries. Validation in other populations will be required. We also acknowledge that controls included in GR@ACE are younger than cases and some of the controls might still develop AD later in life. This fact does not invalidate the analysis although reported estimates must be considered conservative. The differences in risk and AAO determined by the PRS of AD are relevant for design clinical trials that over-represent $A P O E \& 4$ carriers, as $A P O E \varepsilon 4$ heterozygous with highest-PRS values have a similar risk and AAO to APOE $\varepsilon 4$ homozygotes (Fig. 5b). These represents $\sim 1 \%$ of our control population, which is the same percentage as all $A P O E \& 4$ homozygotes. A trial that aims to include APOE $\varepsilon 4$ homozygotes, could consider widening the selection criteria and in this way hasten the enrollment process. Also, our PRS could aid at the interpretation of the results of clinical trials, as it determines a relevant proportion of the $\mathrm{AAO}$, which could either mimic or obscure a treatment effect.

The most interesting finding from our GWAS is the discovery of a common protective $(\mathrm{MAF}(\mathrm{C}$-allele $)=0.483)$ intronic variant in the $A P P$ gene. Our results directly support $A P P$ production or processing as a causal pathway not only in familial $\mathrm{AD}$ but in common sporadic AD. The SNP is in a DNase hypersensitive area of $295 \mathrm{bp}$ (chr21:27473781-27474075) possibly involved in the transcriptional regulation of the APP gene. rs2154481 is an eQTL for the $A P P$ mRNA and an antisense transcript of the APP gene named AP001439.2 in public eQTL databases ${ }^{25}$ (Supplementary Fig. 4). Functional evidence supports a modified $A P P$ transcription $^{26}$ as an LD block of 13 SNPs within the APP locus 


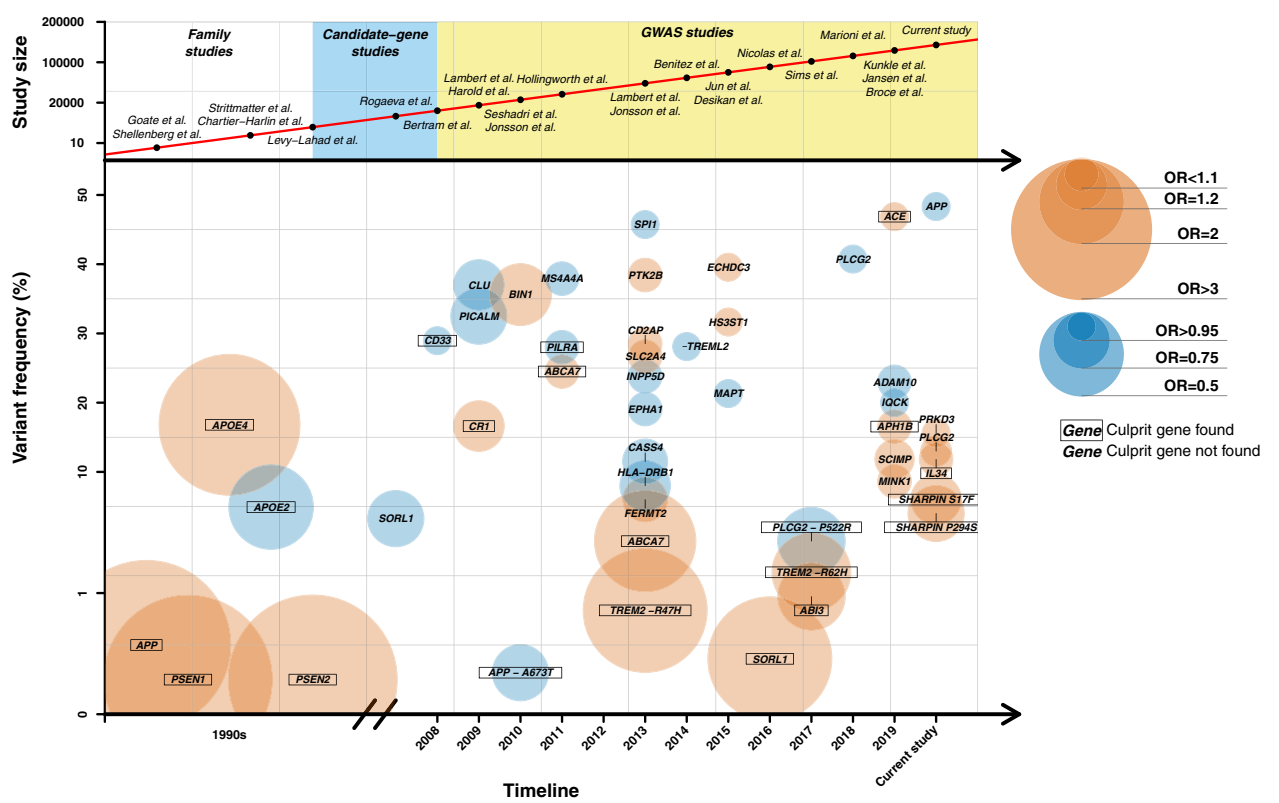

Fig. 3 Genetic landscape for Alzheimer's disease. This figure shows the history of genetic discoveries in AD research over the past 30 years. This figure was constructed to our best knowledge of literature, but is not a systematic review of literature. For common variants, we selected only signals firmly replicated in large meta-GWAS (Lambert et al. ${ }^{3}$, Kunkle et al. ${ }^{20}$, Jun et al. ${ }^{43}$, Sims et al. ${ }^{7}$, Jansen et al. ${ }^{38}$ and present study). For rare variants, we only selected those variants widely replicated excluding those loci presenting conflicting results. Abbreviations and more information about the genes can be found in Supplementary Data 4. The risk alleles associated with AD were represented in orange and the protective alleles in blue. GWAS Genome-Wide Association Study, OR odds ratio.

(including rs2154481) increased the TFCP2 transcription factor avidity to its binding site and increased the enhancer activity of this specific intronic region ${ }^{26}$. Based on this evidence, we can postulate that a life-long slightly higher $A P P$ gene expression protects the brain from $\mathrm{AD}$ insults. Still, this seems counterintuitive as duplications of the gene lead to early-onset $\mathrm{AD}^{27}$. A $\mathrm{U}$-shaped effect, or hormesis effect of $A P P$ might help explain our observations and it might also fit the accelerated cognitive deterioration observed in $\mathrm{AD}$ patients treated with beta-secretase inhibitors $^{28,29}$ as these reduce beta-amyloid in their brain. An alternative hypothesis is that mechanisms underlying the variant are related to the overexpression of protective fragments of the APP protein ${ }^{30}$. Disentangling the molecular mechanism of our finding will help refine and steer the amyloid hypothesis.

Additionally, other three variants identified are altering protein sequence or affecting regulatory motifs. Two independent missense mutations in SHARPIN increased the AD risk. SHARPIN was previously proposed as an $\mathrm{AD}$ candidate gene $\mathrm{e}^{31,32}$, and functional analysis of a rare missense variant (NM_030974.3:p. Gly186Arg) resulted in the aberrant cellular localization of the variant protein and attenuated the activation of NF- $\kappa \mathrm{B}$, a central mediator of inflammatory and immune responses. Functional analysis of the two identified missense variants will show if the effect on immune reaction in $\mathrm{AD}$ is similar. The variant located in the CHRNE which encodes a subunit of the cholinergic receptor (AChR) is a strong modulator of CHRNE expression. The same allele that increases $\mathrm{AD}$ risk increases the expression in the brain and other tissues according to GTEx $\left(p=2.1 \times 10^{-13}\right)$ (Supplementary Fig. 5). The detection of a potential hypermorph allele linked to AD risk and affecting cholinergic function could reintroduce this neurotransmitter pathway into the search for preventative strategies. Further functional studies are needed to consolidate this hypothesis.

Altogether, we described six additional loci associated with sporadic $\mathrm{AD}$. These signals reinforce that $\mathrm{AD}$ is a complex disease in which amyloid processing and immune response play key roles. We add to the growing body of evidence that the polygenic scores of all genetic loci to date, in combination with $A P O E$ genotypes, are robust tools that are associated with $\mathrm{AD}$ and its AAO. These properties make PRS promising in selecting individuals at risk to apply preventative therapeutic strategies.

\section{Methods}

Data. Participants in this study were obtained from multiple sources, including raw data from case-control samples collected by GR@ACE/DEGESCO, summary statistics data from the case-control samples in the IGAP and the summary statistics of AD-by-proxy phenotype from the UK Biobank. An additional case-control samples from 16 independent cohorts (19,087 AD cases and 39,101 controls) was used for replication, largely collected and analyzed by the European Alzheimer's Disease Biobank (JPND-EADB) project. Full descriptions of the samples and their respective phenotyping and genotyping procedures are provided in the Supplementary Methods.

GR@ACE. The GR@ACE study ${ }^{19}$ recruited AD patients from Fundació ACE Institut Català de Neurociències Aplicades (Catalonia, Spain), and control individuals from three centers: Fundació ACE (Barcelona, Spain), Valme University Hospital (Seville, Spain), and the Spanish National DNA Bank-Carlos III (University of Salamanca, Spain) (http://www.bancoadn.org). Additional cases and controls were obtained from dementia cohorts included in the Dementia Genetics Spanish Consortium (DEGESCO) ${ }^{33}$. At all sites, AD diagnosis was established by a multidisciplinary working group-including neurologists, neuropsychologists, and social workers-according to the DSM-IV criteria for dementia and the National Institute on Aging and Alzheimer's Association's (NIA-AA) 2011 guidelines for diagnosing $\mathrm{AD}$. In our study, we considered as $\mathrm{AD}$ cases any individuals with dementia diagnosed with probable or possible $\mathrm{AD}$ at any point in their clinical course. For further details on the contribution of the sites, see Supplementary Data 10. Written informed consent was obtained from all the participants. The ethics and scientific committees have approved this research protocol (Acta 25/ 2016, Ethics Committee H., Clinic I Provincial, Barcelona, Spain).

Genotyping, quality control, and imputation. DNA was extracted from peripheral blood according to standard procedures using the Chemagic system (Perkin Elmer). Samples reaching DNA concentrations of $>10 \mathrm{ng} / \mu \mathrm{l}$ and presenting high integrity were included for genotyping. Cases and controls were randomized across sample plates to avoid batch effects.

Genotyping was conducted using the Axiom 815K Spanish biobank array (Thermo Fisher) at the Spanish National Center for Genotyping (CeGEN, Santiago de Compostela, Spain). The genotyping array not only is an adaptation of the Axiom biobank genotyping array but also contains rare population-specific 
a

Clinical AD

All studies $p<5 e-8$

Excluding novel

Kunkle 2019 p $<1$ e-7

Kunkle 2019 p $<1$ e-6

Kunkle $2019 \mathrm{p}<1 \mathrm{e}-5$

Kunkle $2019 \mathrm{p}<1 \mathrm{e}-4$

Kunkle $2019 \mathrm{p}<1 \mathrm{e}-3$

Kunkle $2019 \mathrm{p}<1 \mathrm{e}-2$

Pathologic AD

All studies $p<5 e-8$

Excluding novel

Kunkle $2019 \mathrm{p}<1 \mathrm{e}-7$

Kunkle $2019 \mathrm{p}<1 \mathrm{e}-6$

Kunkle 2019 p $<1 e-5$

Kunkle $2019 p<1 \mathrm{e}-4$

Kunkle $2019 \mathrm{p}<1 \mathrm{e}-3$

Kunkle 2019 p $<1 \mathrm{e}-2$

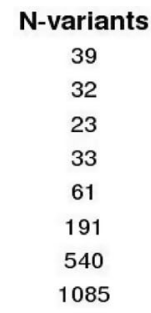

N-variants

39
32
23
33
61
191
540
1085

\title{
b
}

\author{
Concomitant pathologies: \\ Only AD pathology \\ Cerebrovascular disease \\ Lewy body disease \\ Microscopic vascular lesions \\ TDP43 aggregates \\ Hippocampal sclerosis
}

Number of concomitant pathologies:

$A D+$ single pathology

$A D+$ two pathologies

$A D+$ three and more pathologies

pathologies:

\section{Sex: \\ Male}

Female

N-cases
320
688

Age at onset
$<65$ years
$65-70$ years
$70-75$ years
$75-80$ years
$80-85$ years
$>85$ years

N-cases
62
81
144
271
289
201

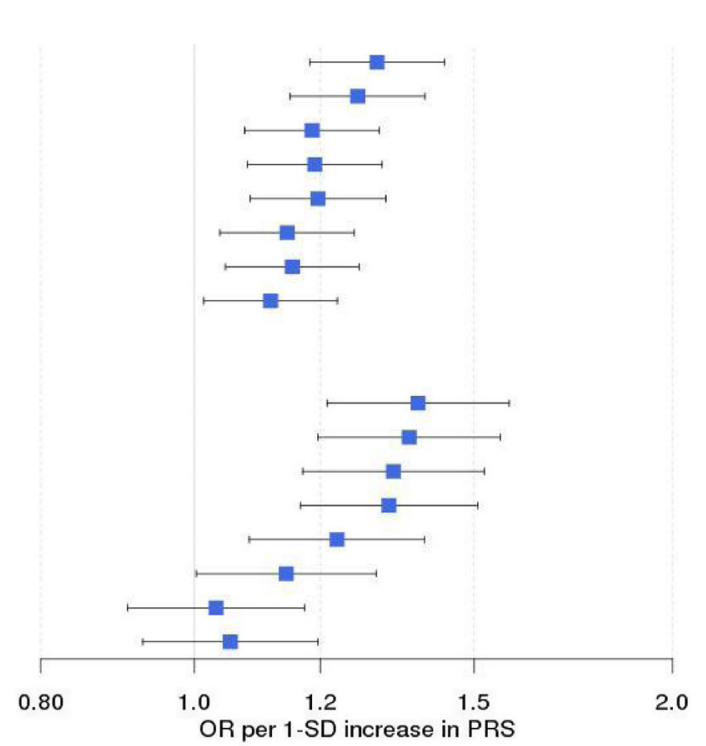

OR $[95 \% \mathrm{Cl}]$

P-value

1.30 [1.18-1.44]

$1.27[1.15-1.40]$

1.19 [1.08-1.31]

$1.19[1.08-1.31]$

$1.20[1.08-1.32]$

$1.14[1.04-1.26]$

$1.15[1.05-1.27]$

1.12 [1.01-1.23]

1.1e-07

2. $1 \mathrm{e}-06$

6. $2 \mathrm{e}-04$

4.7e-04

3. $6 \mathrm{e}-04$

6. $9 e-03$

4.1e-03

2.5e-02

\section{OR $[95 \% \mathrm{Cl}]$}

P-value

1.38 [1.21-1.58]

1.37 [1.20-1.56]

1.33 [1.17-1.52]

1.33 [1.17-1.51]

$1.23[1.08-1.40]$

1.14 [1.00-1.30]

1.03 [0.91-1.17]

1.05 [0.93-1.20]

1.5e-06

4.0e-06

$1.7 \mathrm{e}-05$

$1.7 e-05$

$1.5 e-03$

4.6e-02

6.3e-01

4. $2 \mathrm{e}-01$

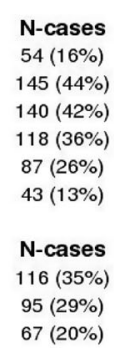

$54(16 \%)$

145 (44\%)

140 (42\%)

$3(13 \%)$

$\mathrm{N}$-cases

$95(29 \%)$

$67(20 \%)$

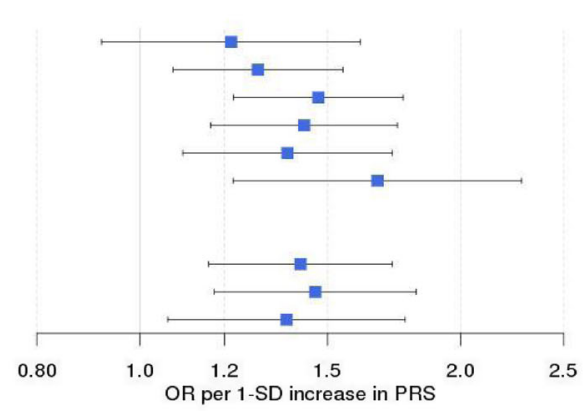

N-controls

379

1007

N-controls
1386
1386
1386
1386
1386
1386

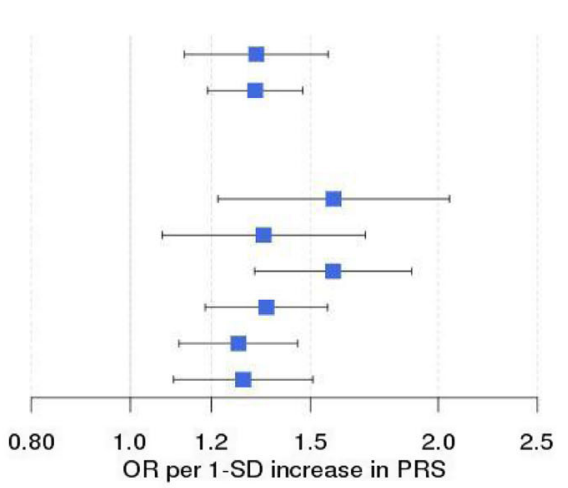

OR $[95 \% \mathrm{Cl}]$

1.22 [0.92-1.61]

1.29 [1.07-1.55]

$1.47[1.22-1.77]$

$1.43[1.17-1.74]$

1.38 [1.10-1.72]

1.67 [1.22-2.28]

OR [ $95 \% \mathrm{Cl}]$

1.41 [1.16-1.73]

1.46 [1.17-1.82]

1.37 [1.06-1.77]
P-value

1.7e-01

6.6e-03

3.7e- 05

5.7e-04

5.8e-03

1.3e-03

P-value

6.1e-04

6.7e-04

1.6e-02

$\begin{array}{cc}\text { OR }[95 \% \mathrm{CI}] & \text { P-value } \\ 1.33[1.13-1.56] & 5.8 \mathrm{e}-04 \\ 1.32[1.19-1.47] & 2.5 e-07 \\ & \\ \text { OR [95\% CI] } & \text { P-value } \\ 1.58[1.22-2.05] & 5.8 e-04 \\ 1.35[1.07-1.70] & 1.0 e-02 \\ 1.58[1.32-1.88] & 4.1 e-07 \\ 1.36[1.18-1.56] & 1.3 e-05 \\ 1.28[1.12-1.46] & 3.5 e-04 \\ 1.29[1.10-1.51] & 1.5 e-03\end{array}$

Fig. 4 Polygenic risk scores for AD. a The 39-SNP PRS association with clinical ( $\mathrm{OR}=1.30,95 \% \mathrm{Cl}[1.18-1.44], p=1.1 \times 10^{-7}$ ) and pathologically confirmed $A D$ cases $\left(O R=1.38\right.$, per $1-S D$ increase in the PRS, $\left.95 \% \mathrm{Cl}[1.21-1.58], p=1.5 \times 10^{-6}\right)$ from EADB-F.ACE/BBB dataset. $\mathbf{b}$ PRS association with AD in the presence of concomitant brain pathologies (besides AD). $\mathbf{c}$ PRS association with AD stratified by sex and AAO. A similar association of the PRS with AD was found in both sexes $\left(\mathrm{OR}_{\text {males }}=1.33,[1.13-1.56], p=5.8 \times 10^{-4}\right.$ vs. $\left.\mathrm{OR}_{\text {females }}=1.32,[1.19-1.47], p=2.5 \times 10^{-7}\right)$. In (a-c) data are presented as Odds Ratio per 1-SD increase in PRS $(95 \% \mathrm{Cl})$. The generated PRS was validated using logistic regression adjusted by four principal components.

variations observed in the Spanish population. The DNA samples were genotyped according to the manufacturer's instructions (Axiom ${ }^{\mathrm{TM}} 2.0$ Assay Manual

Workflow). The Axiom 2.0 assay interrogates biallelic SNPs and simple indels in a single-assay workflow. Starting with $200 \mathrm{ng}$ of genomic DNA, the samples were processed through a manual target preparation protocol, followed by automated processing of the array plates in the GeneTitan Multi-Channel (MC) instrument. Target preparation involved DNA amplification, fragmentation, purification, and resuspension of the target in a hybridization cocktail. The hyb-ready targets were then transferred to the GeneTitan MC instrument for automated, hands-free processing, including hybridization, staining, washing, and imaging. The CEL files were generated using the GeneTitan MC instrument. Quality control (QC) was performed for samples and plates using the Affymetrix power tool (APT) 1.15.0 software following the Axiom data analysis workflow. The sample quality was determined based on the resolution of AT and GC channels in a group of nonpolymorphic SNPs (resolution $>0.82$ ). Samples with a call rate greater than $97 \%$ and plates with an average call rate above $98.5 \%$ were included for final SNP calling. The samples were jointly called. Markers passing all the QC tests were used in downstream analysis $\left(N_{\mathrm{SNP}}=729,868 ; 95.4 \%\right)$ using the SNPolisher R package (Thermo Fisher). To assess the sample genotyping concordance, we intentionally resampled 200 samples and determined a concordance rate of $99.5 \%$.

We also conducted previously described standard QC prior to imputation ${ }^{19}$. In brief, individual QC includes genotype call rates $>97 \%$, sex checks, and no excess heterozygosity; we removed population outliers as well (European cluster of 1000 Genomes). We included variants with a call rate of $>95 \%$, with a minor allele frequency (MAF) of $>0.01$, in Hardy-Weinberg equilibrium $\left(p<1 \times 10^{-4}\right.$ in controls) and without differential missingness between cases and controls 
a

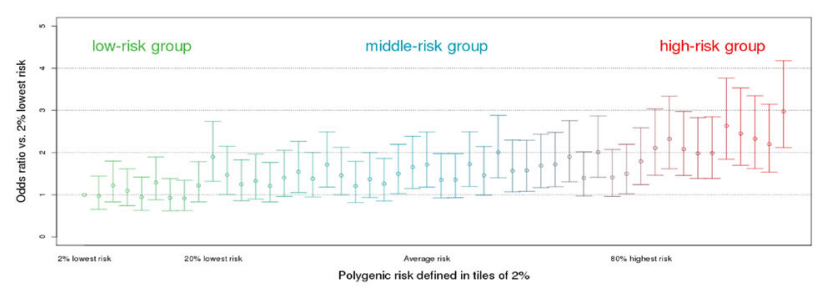

C

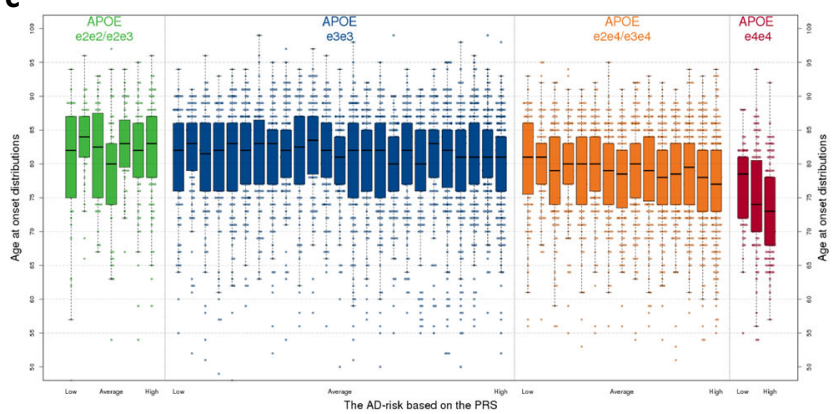

b

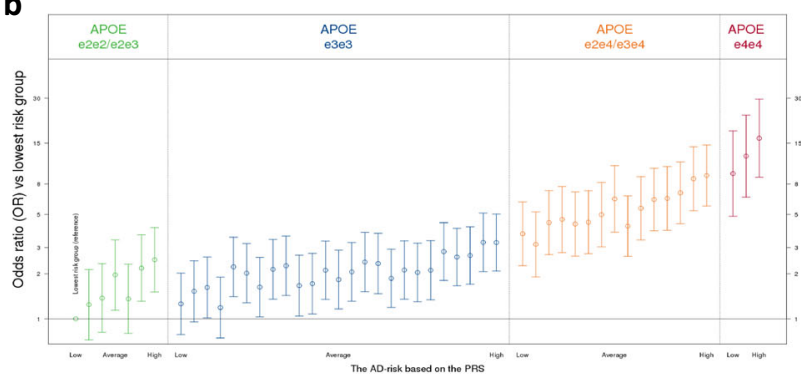

d

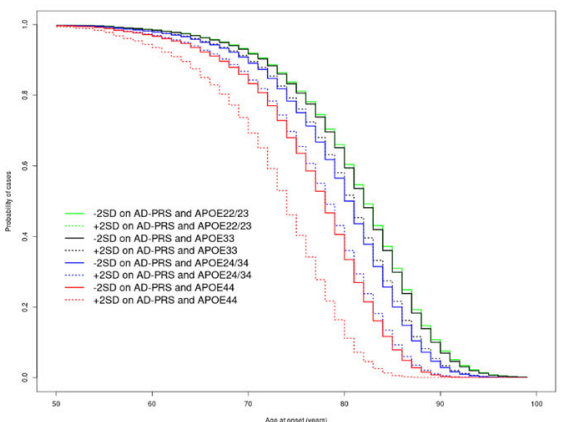

Fig. 5 Polygenic Risk Scores APOE stratification for AD in $\boldsymbol{n}=12,386$ biologically independent samples from GR@ACE/DEGESCO. a The AD risk of PRS groups compared to those with the $2 \%$ lowest risk. The $2 \%$ highest risk had a 3.0 -fold ( $95 \% \mathrm{Cl}[2.12-4.18], p=3.2 \times 10^{-10}$ ) increased risk compared with those with the $2 \%$ lowest risk. No interaction was found between the PRS and APOE genotypes ( $p$ value $=0.76$ ). $\mathbf{b}$ The AD risk stratified by PRS and APOE risk groups compared to the lowest risk group (OR $95 \% \mathrm{CI}$ ). Association was found between highest and lowest-PRS percentiles within the APOE genotype groups: $\varepsilon 2 \varepsilon 2 / \varepsilon 2 \varepsilon 3$ carriers ( $O R=2.48$ [1.51-4.08], $\left.p=3.4 \times 10^{-4}\right), \varepsilon 3 \varepsilon 3$ carriers $\left(\mathrm{OR}=2.67\right.$ [1.93-3.69], $\left.p=3.5 \times 10^{-9}\right), \varepsilon 2 \varepsilon 4 / \varepsilon 3 \varepsilon 4$ carriers $(\mathrm{OR}=2.47$ [1.67-3.66], $\left.p=6.8 \times 10^{-6}\right)$, and $\varepsilon 4 \varepsilon 4$ carriers $\left(O R=2.02[1.05-3.85], p=3.4 \times 10^{-2}\right)$. Comparisons of the highest and lowest-PRS percentiles with respect to the APOE genotype groups: a difference was found between highest $\varepsilon 2 \varepsilon 2 / \varepsilon 2 \varepsilon 3$ carriers vs. lowest $\varepsilon 3 \varepsilon 3$ carriers $(\mathrm{OR}=0.51$ [0.34-0.75], $\left.p=7.8 \times 10^{-4}\right)$, but not between highest $\varepsilon 3 \varepsilon 3$ carriers vs. lowest $\varepsilon 2 \varepsilon 4 / \varepsilon 3 \varepsilon 4$ carriers $(\mathrm{OR}=1.17$ [0.82-1.66], $p=0.40)$ and highest $\varepsilon 2 \varepsilon 4 / \varepsilon 3 \varepsilon 4$ carriers vs. lowest $\varepsilon 4 \varepsilon 4$ carriers $(O R=0.89$ [0.52-1.53], $p=0.68)$. c The AAO of AD stratified by PRS and APOE risk groups. No difference in odds for AD was found between the PRS percentiles with AAO in APOE $\varepsilon 2 \varepsilon 2 / \varepsilon 2 \varepsilon 3$ (lowest $=82$ years, highest $=83$ years, $\left.p_{\text {Wilcoxon }}=0.39\right)$ and $A P O E \varepsilon 3 \varepsilon 3$ (lowest $=82$ years, highest $=81$ years, $p=0.16$ ). However, a 4-year difference was found between APOE $\varepsilon 4$ heterozygotes $\left(p_{\text {Wilcoxon }}=6.9 \times 10^{-5}, 81\right.$ years compared with 77 years) and 5.5 years difference ( $p_{\text {Wilcoxon }}=4.6 \times 10^{-5}, 78.5$ years compared with 73 years) in APOE $\varepsilon 4$ homozygotes. Data are represented as boxplots as described in the manual of ggplot2 package in R. a-c Logistic regression models adjusted for four population ancestry components were used as statistical test. $\mathbf{d}$ Cox regression model on AAO. The determinants are the PRS and the APOE categories, a PRS ${ }^{\star} A P O E$ interaction term and population substructure as covariates. The curve shows the probability a case in one of the eight groups has developed AD by a certain age ( $x$-axis).

(Supplementary Data 11, Supplementary Fig. 1). Imputation was carried out using the Haplotype reference consortium ${ }^{34}$ (HRC, full panel) and the 1000 Genomes reference panel ${ }^{35}$ (for indels only) on the Michigan Imputation Server (https:// imputationserver.sph.umich.edu). Rare variants $(\mathrm{MAF}<0.001)$ and variants with low imputation quality $\left(R^{2}<0.30\right)$ were excluded. Logistic regression models, adjusted for the first four ancestry principal components ${ }^{19}$, were fitted using Plink (v2.00a). Population-based controls were used; therefore, age was not included as a covariate. Age and gender statistically behave like phenotype proxies (for AD status in this case). Therefore, adjusting for co-variation with age and gender could result in an over-adjustment of GWAS results. After QC steps, we included 6,331 AD cases and 6,055 control individuals and tested 14,542,816 genetic variants for association with $\mathrm{AD}$.

IGAP summary statistics. The GWAS summary results from the IGAP were downloaded from the National Institute on Aging Genetics of Alzheimer's Disease Data Storage Site (NIAGADS, https://www.niagads.org/) ${ }^{20}$. Details on data generation and analyses by the IGAP have been previously described ${ }^{20}$. In brief, the IGAP is a large study based upon genome-wide association using individuals of European ancestry. Stage 1 of the IGAP comprises 21,982 AD cases and 41,944 cognitively normal controls from four consortia: the Alzheimer Disease Genetics Consortium (ADGC), the European Alzheimer's Disease Initiative (EADI), the Cohorts for Heart and Aging Research in Genomic Epidemiology (CHARGE) Consortium, and the Genetic and Environmental Risk in AD/Defining Genetic, Polygenic, and Environmental Risk for Alzheimer's Disease (GERAD/PERADES) Consortium. Summary statistics are available for 11,480,632 variants, both genotyped and imputed (1000 Genomes phase 1, v3). In Stage 2, 11,632 SNPs were genotyped in an independent set of $8362 \mathrm{AD}$ cases and 10,483 controls.

UK Biobank summary statistics. UK Biobank data-including health, cognitive, and genetic data-was collected on over 500000 individuals aged 37-73 years from across Great Britain (England, Wales, and Scotland) at the study baseline
(2006-2010) (http://www.ukbiobank.ac.uk) ${ }^{36}$. Several groups have demonstrated the utility of self-report of parental history of AD for case ascertainment in GWAS (proxy-AD approach) $)^{21,37,38}$. For this study, we used the published summary statistics of Marioni et al. ${ }^{21}$. They included, after stringent QC, 314,278 unrelated individuals for whom $\mathrm{AD}$ information was available on at least one parent in the UK Biobank (https://datashare.is.ed.ac.uk/handle/10283/3364). In brief, the 27,696 participants whose mothers had dementia (maternal cases) were compared with the 260,980 participants whose mothers did not have dementia. Likewise, the 14,338 participants whose fathers had dementia (paternal cases) were compared with the 245,941 participants whose fathers did not have dementia ${ }^{21}$. The phenotype of the parents is independent, and therefore, the estimates could be metaanalyzed. After analysis, the effect estimates were made comparable to a casecontrol setting. Further information on the transformation of the effect sizes can be found elsewhere ${ }^{21,39}$. The data available comprises summary statistics of 7,794,553 SNPs imputed to the HRC reference panel (full panel).

Meta-GWAS of AD. After study-specific variant filtering and quality-control procedures, we performed a fixed effects inverse-variance-weighted metaanalysis ${ }^{22}$ on the discovery and follow-up stages (Supplementary Data 1 and Supplementary Data 12). To determine the lead SNPs (those with the strongest association per genomic region), we performed clumping on SNPs with a GWS $p$ value $\left(p<5 \times 10^{-8}\right)$ (Plink v1.90, maximal linkage disequilibrium (LD) with $R^{2}<$ 0.001 and physical distance $250 \mathrm{~Kb}$ ). In the APOE region, we only considered the APOE $\varepsilon 4$ (rs429358) and APOE $\varepsilon 2$ (rs7412) SNPs ${ }^{40}$. LD information was calculated using the GR@ACE imputed genotypes as a reference. Polygenicity and confounding biases, such as cryptic relatedness and population stratification, can yield an inflated distribution of test statistics in GWAS. To distinguish between inflation from a true polygenic signal and bias we quantified the contribution of each by examining the relationship between test statistics and linkage disequilibrium (LD) using the LD Score regression intercept (LDSC software ${ }^{41}$ ). Chromosomal regions associated with $\mathrm{AD}$ in previous studies were excluded from follow-up (Lambert 
et al. ${ }^{3}$, Kunkle et al. ${ }^{42}$, and Jansen et al. ${ }^{38}$ ). We tested all variants with suggestive association $\left(p<10^{-5}\right)$ located in proximity $(200 \mathrm{~kb})$ of genomic regions selected for follow-up to allow for the potential refinement of the top associated variant.

Conditional analyses were performed in regions where multiple variants were associated with $\mathrm{AD}$ using logistic regression models, adjusting for the genetic variants in the region (Supplementary Data 13,14).

Regional plots were generated with a mixture of homemade Python (v2.7) and $R$ (v3.6.0) scripts. Briefly, given an input variant, we calculated the LD between the input variant and all the surrounding variants within a window of length defined by the user. The LD was calculated in the 1000 Genomes samples of European ancestry. We used gene positions from RefSeq (release 93); in the case of multiple gene models for a given gene, we reported the model with the largest number of exons. We used recombination rates from HapMap II and chromatin states from ENCODE/Broad (15 states were grouped to highlight the predicted functional elements). As a reference genome, we used GRCh37. Quantile-quantile plots, Manhattan plots, and the exploration of genomic inflation factors were performed using the R package qqman.

Polygenic risk scores. We calculated a weighted individual PRS based on the 39 genetic variants that showed GWS evidence of association with $\mathrm{AD}$ in the present study, excluding $A P O E$ to check the impact of PRS modulating APOE risk (Table 1 and Supplementary Data 3 ). The selected variants were directly genotyped or imputed with high quality (median imputation score $R^{2}=0.93$ ). The PRSs were generated by multiplying the genotype dosage of each risk allele for each variant by its respective weight and then summing across all variants. We weighted this by the effect size from previous IGAP studies [Kunkle et al. ${ }^{42}$ (36 variants), Sims et al. ${ }^{7}$ (2 variants), Jun et al. ${ }^{43}$ (MAPT locus), Supplementary Data 5]. The generated PRS was validated using logistic regression adjusted by four principal components in a sample of $676 \mathrm{AD}$ cases diagnosed based on clinical criteria and 332 pathologically confirmed $\mathrm{AD}$ cases from the European Alzheimer's Disease Biobank-Fundació ACE/Barcelona Brain Bank dataset (EADB-F.ACE/BBB, Supplementary Information). This dataset was not used in prior genetic studies. In this dataset, all pathologically confirmed cases were scored for the presence or absence of concomitant pathologies. In all analyses, we compared the AD patients to the same control dataset $(n=1386)$. We performed analyses to test the robustness of the PRS. We tested the effect of adding variants below the genome-wide significance threshold using a pruning and thresholding approach. For this, we used the summary statistics of the IGAP ${ }^{42}$ study, and we selected independent variants using the clump_data() function from the TwoSampleMR package (v0.4.25). We used strict settings for clumping $\left(R^{2}=0.001\right.$ and window $\left.=1 \mathrm{MB}\right)$ and increasing $\mathrm{p}$ value thresholds $\left(>1 \times 10^{-7},>1 \times 10^{-6},>1 \times 10^{-5},>1 \times 10^{-4},>1 \times 10^{-3}\right.$, and $>1 \times$ $10^{-2}$ ). We tested the association of the results with clinically diagnosed and pathologically confirmed $\mathrm{AD}$ patients. To evaluate the effect of diagnostic certainty, we tested whether the PRS was different between the two patient groups. For the PRS with 39 GWS variants, we tested whether the PRS had sex-specific effects, whether it resulted in different age-of-onset groups of $\mathrm{AD}$, and the effect of the PRS in the presence of concomitant brain pathologies.

Risk stratification of the validated PRSs. We searched for the groups at the highest risk of AD in the GR@ACE dataset (6331 AD cases and 6055 controls). We stratified the population into PRS percentiles, taking into account survival bias anticipated at old age ${ }^{18}$. To eliminate selection bias, we calculated the boundaries of the percentiles in the control participants aged 55 years and younger $(n=3546)$. Based on the boundaries from this population, the rest of the controls and all $\mathrm{AD}$ cases were then assigned into their appropriate percentiles. We first explored risk stratification using only the PRSs. For this, we split the PRSs into 50 groups (2 percentiles) and compared all groups with that which had the lowest PRS. Second, we explored risk stratification considering both the $A P O E$ genotypes and the PRSs. The APOE genotypes were pooled in the analyses as APOE $\varepsilon 2 \varepsilon 2 / \varepsilon 2 \varepsilon 3(n=998$, split into 7 PRS groups), APOE $\varepsilon 3 \varepsilon 3$ ( $n=7611$, split into 25 PRS groups), $A P O E$ $\varepsilon 2 \varepsilon 4 / \varepsilon 3 \varepsilon 4$ ( $n=3399$, split into 15 PRS groups), and APOE $\varepsilon 4 \varepsilon 4$ ( $n=382$, split into 3 PRS groups). We studied the effect of PRS across groups of individuals stratified by the APOE genotypes with the lowest-PRS group ( $A P O E$ as the reference group using logistic regression models adjusted for four population ancestry components). Finally, we compared the median AAO using a Wilcoxon test.

We implemented a Cox regression model on AAO in the GR@ACE/DEGESCO dataset case-only adjusted for covariates as $A P O E$ group, the interaction between the PRS and $A P O E$ and four population ancestry components. All analyses were done in $\mathrm{R}$ (v3.4.2).

Functional annotation. We used Functional Mapping and Annotation of GenomeWide Association Studies ${ }^{23}$ (FUMA, v1.3.4c) to interpret SNP-trait associations (see Supplementary Methods and Supplementary Data 15-18). FUMA is an online platform that annotates GWAS findings and prioritizes the most likely causal SNPs and genes using information from 18 biological data repositories and tools. As input, we used the summary statistics of our meta-GWAS. Gene prioritization is based on a combination of positional mapping, expression quantitative trait loci (eQTL) mapping, and chromatin interaction mapping. Functional annotation was performed by applying a methodology similar to that described by Jansen et al. ${ }^{38}$. We referred to the original publication for details on the methods and repositories of FUMA ${ }^{23}$.
Reporting summary. Further information on research design is available in the Nature Research Reporting Summary linked to this article.

\section{Data availability}

The discovery summary statistics of this study are publicly available in Fundació ACE server [https://fundacioace-my.sharepoint.com/:u:/g/personal/iderojas_fundacioace_org/ EaTwlPg9cRJHn7Kos4h39OUBaxajsjJHL_C110fC89bc8w?e=ZdcEUy].

Received: 17 July 2020; Accepted: 17 March 2021; Published online: 07 June 2021

\section{References}

1. Pericak-Vance, M. A. et al. Linkage studies in familial Alzheimer disease: evidence for chromosome 19 linkage. Am. J. Hum. Genet. 48, 1034-1050 (1991).

2. Seshadri, S. et al. Genome-wide analysis of genetic loci associated with Alzheimer disease. JAMA J. Am. Med. Assoc. 303, 1832-1840 (2010).

3. Lambert, J. C. et al. Meta-analysis of 74,046 individuals identifies 11 new susceptibility loci for Alzheimer's disease. Nat. Genet. 45, 1452-1458 (2013).

4. Jonsson, T. et al. Variant of TREM2 associated with the risk of Alzheimer's disease. N. Engl. J. Med. 368, 107-116 (2013).

5. Guerreiro, R. et al. TREM2 variants in Alzheimer's disease. N. Engl. J. Med. 368, 117-127 (2013).

6. Jonsson, T. et al. A mutation in APP protects against Alzheimer's disease and age-related cognitive decline. Nature 488, 96-99 (2012).

7. Sims, R. et al. Rare coding variants in PLCG2, ABI3, and TREM2 implicate microglial-mediated innate immunity in Alzheimer's disease. Nat. Genet. 49, 1373-1384 (2017).

8. Ridge, P. G. et al. Assessment of the genetic variance of late-onset Alzheimer's disease. Neurobiol. Aging 41, 200.e13-200.e20 (2016).

9. 2020 Alzheimer's disease facts and figures. Alzheimer's Dement. 16 391-460 (2020).

10. Reiman, E. M. et al. Alzheimer's prevention initiative: a plan to accelerate the evaluation of presymptomatic treatments. J. Alzheimer's Dis 26, 321-329 (2011).

11. Huang, L.-K., Chao, S.-P. \& Hu, C.-J. Clinical trials of new drugs for Alzheimer disease. J. Biomed. Sci. 27, 18 (2020).

12. Rodríguez-Rodríguez, E. et al. Genetic risk score predicting accelerated progression from mild cognitive impairment to Alzheimer's disease. J. Neural Transm. 120, 807-812 (2013).

13. Adams, H. H. H. et al. Genetic risk of neurodegenerative diseases is associated with mild cognitive impairment and conversion to dementia. Alzheimer's Dement. https://doi.org/10.1016/j.jalz.2014.12.008 (2015).

14. Desikan, R. S. et al. Genetic assessment of age-associated Alzheimer disease risk: Development and validation of a polygenic hazard score. PLoS Med. https://doi.org/10.1371/journal.pmed.1002258 (2017).

15. Lacour, A. et al. Genome-wide significant risk factors for Alzheimer's disease: role in progression to dementia due to Alzheimer's disease among subjects with mild cognitive impairment. Mol. Psychiatry 22, 153-160 (2016).

16. Sleegers, K. et al. A 22-single nucleotide polymorphism Alzheimer's disease risk score correlates with family history, onset age, and cerebrospinal fluid $\mathrm{A} \beta$ 42. Alzheimer's Dement 11, 1452-1460 (2015).

17. Zhang, Q. et al. Risk prediction of late-onset Alzheimer's disease implies an oligogenic architecture. Nat. Commun. https://doi.org/10.1038/s41467-02018534-1 (2020).

18. van der Lee, S. J. et al. The effect of APOE and other common genetic variants on the onset of Alzheimer's disease and dementia: a community-based cohort study. Lancet Neurol. https://doi.org/10.1016/S1474-4422(18)30053-X (2018).

19. Moreno-Grau, S. et al. Genome-wide association analysis of dementia and its clinical endophenotypes reveal novel loci associated with Alzheimer's disease and three causality networks: The GR@ACE project. Alzheimer's Dement. 0, 1333-1347 (2019).

20. Kunkle, B. W. et al. Genetic meta-analysis of diagnosed Alzheimer's disease identifies new risk loci and implicates AmyloidBeta, tau, immunity and lipid processing. Nat. Genet. 51, 414-430 (2019).

21. Marioni, R. E. et al. GWAS on family history of Alzheimer's disease. Transl. Psychiatry https://doi.org/10.1038/s41398-018-0150-6 (2018).

22. Willer, C. J., Li, Y. \& Abecasis, G. R. METAL: Fast and efficient meta-analysis of genomewide association scans. Bioinformatics https://doi.org/10.1093/ bioinformatics/btq340 (2010) .

23. Watanabe, K., Taskesen, E., van Bochoven, A. \& Posthuma, D. Functional mapping and annotation of genetic associations with FUMA. Nat. Commun. https://doi.org/10.1038/s41467-017-01261-5 (2017).

24. Khera, A. V. et al. Genome-wide Polygenic Scores for Common Diseases Identify Individuals With Risk Equivalent to Monogenic Mutations. Nat. Genet. 50, 1219-1224 (2018). 
25. Lonsdale, J. et al. The genotype-tissue expression (GTEx) project. Nat. Genet. 45, 580-585 (2013)

26. Myrum, C., Nikolaienko, O., Bramham, C. R., Haavik, J. \& Zayats, T. Implication of the APP gene in intellectual abilities. J. Alzheimer's Dis 59, 723-735 (2017).

27. Cenini, G. et al. Wild type but not mutant APP is involved in protective adaptive responses against oxidants. Amino Acids 39, 271-283 (2010).

28. Egan, M. F. et al. Randomized trial of verubecestat for mild-to-moderate Alzheimer's disease. N. Engl. J. Med. 378, 1691-1703 (2018).

29. Panza, F., Lozupone, M. \& Watling, M. \& Imbimbo, B. P. Do BACE inhibitor failures in Alzheimer patients challenge the amyloid hypothesis of the disease? Expert Rev. Neurother. 19, 599-602 (2019).

30. Hefter, D. \& Draguhn, A. APP as a protective factor in acute neuronal insults. Front. Mol. Neurosci. 10, 22 (2017)

31. Lancour, D. et al. One for all and all for One: Improving replication of genetic studies through network diffusion. PLoS Genet 14, e1007306 (2018).

32. Asanomi, Y. et al. A rare functional variant of SHARPIN attenuates the inflammatory response and associates with increased risk of late-onset Alzheimer's disease. Mol. Med. 25, 20 (2019).

33. Ruiz, A. et al. Assessing the role of the TREM2 p.R47H variant as a risk factor for Alzheimer's disease and frontotemporal dementia. Neurobiol. Aging 35, 444.e1-444.e4 (2014)

34. McCarthy, S. et al. A reference panel of 64,976 haplotypes for genotype imputation. Nat. Genet. 48, 1279-1283 (2016).

35. 1000 Genomes Project Consortium. et al. A global reference for human genetic variation. Nature 526, 68-74 (2015).

36. Sudlow, C. et al. UK Biobank: an open access resource for identifying the causes of a wide range of complex diseases of middle and old Age. PLOS Med. 12, e1001779 (2015).

37. Liu, J. Z., Erlich, Y. \& Pickrell, J. K. Case-control association mapping by proxy using family history of disease. Nat. Genet. 49, 325-331 (2017).

38. Jansen, I. E. et al. Genome-wide meta-analysis identifies new loci and functional pathways influencing Alzheimer's disease risk. Nat. Genet. 51, 404-413 (2019).

39. Lloyd-Jones, L. R., Robinson, M. R., Yang, J. \& Visscher, P. M. Transformation of summary statistics from linear mixed model association on all-or-none traits to odds ratio. Genetics 208, 1397-1408 (2018).

40. Jun, G. et al. Comprehensive search for Alzheimer disease susceptibility loci in the APOE region. Arch. Neurol. 69, 1270-1279 (2012).

41. Bulik-Sullivan, B. et al. LD score regression distinguishes confounding from polygenicity in genome-wide association studies. Nat. Genet. https://doi.org/ 10.1038/ng.3211 (2015).

42. Kunkle, B. W. et al. Genetic meta-analysis of diagnosed Alzheimer's disease identifies new risk loci and implicates $A \beta$, tau, immunity and lipid processing. Nat. Genet. 51, 414-430 (2019).

43. Jun, G. et al. A novel Alzheimer disease locus located near the gene encoding tau protein. Mol. Psychiatry 21, 108-117 (2016).

\section{Acknowledgements}

We would like to thank patients and controls who participated in this project. The present work has been performed as part of the doctoral program of I. de Rojas at the Universitat de Barcelona (Barcelona, Spain) supported by national grant from the Instituto de Salud Carlos III FI20/00215. The Genome Research @ Fundació ACE project (GR@ACE) is supported by Grifols SA, Fundación bancaria "La Caixa", Fundació ACE, and CIBERNED. A.R. and M.B. receive support from the European Union/EFPIA Innovative Medicines Initiative Joint undertaking ADAPTED and MOPEAD projects (grant numbers 115975 and 115985, respectively). M.B. and A.R. are also supported by national grants PI13/02434, PI16/01861, PI17/01474, PI19/01240 and PI19/01301. Acción Estratégica en Salud is integrated into the Spanish National R + D + I Plan and funded by ISCIII (Instituto de Salud Carlos III)-Subdirección General de Evaluación and the Fondo Europeo de Desarrollo Regional (FEDER-"Una manera de hacer Europa"). Some control samples and data from patients included in this study were provided in part by the National DNA Bank Carlos III (www.bancoadn.org, University of Salamanca, Spain) and Hospital Universitario Virgen de Valme (Sevilla, Spain); they were processed following standard operating procedures with the appropriate approval of the Ethical and Scientific Committee. Amsterdam dementia Cohort (ADC): Research of the Alzheimer center Amsterdam is part of the neurodegeneration research program of Amsterdam Neuroscience. The Alzheimer Center Amsterdam is supported by Stichting Alzheimer Nederland and Stichting VUmc fonds. The clinical database structure was developed with funding from Stichting Dioraphte. Genotyping of the Dutch case-control samples was performed in the context of EADB (European Alzheimer DNA biobank) funded by the JPco-fuND FP-829-029 (ZonMW project number 733051061). 100-Plus study: We are grateful for the collaborative efforts of all participating centenarians and their family members and/or relations. This work was supported by Stichting Alzheimer Nederland (WE09.2014-03), Stichting Diorapthe, horstingstuit foundation, Memorabel (ZonMW project number 733050814, 733050512) and Stichting VUmc Fonds. Genotyping of the 100-Plus Study was performed in the context of EADB (European Alzheimer DNA biobank) funded by the JPco-fuND FP-829-029 (ZonMW project number
733051061). Longitudinal Aging Study Amsterdam (LASA) is largely supported by a grant from the Netherlands Ministry of Health, Welfare and Sports, Directorate of LongTerm Care. The authors are grateful to all LASA participants, the fieldwork team and all researchers for their ongoing commitment to the study. This work was supported by a grant (European Alzheimer DNA BioBank, EADB) from the EU Joint Program-Neurodegenerative Disease Research (JPND) and also funded by Inserm, Institut Pasteur de Lille, the Lille Métropole Communauté Urbaine, the French government's LABEX DISTALZ program (development of innovative strategies for a transdisciplinary approach to $\mathrm{AD}$ ). Genotyping of the German case-control samples was performed in the context of EADB (European Alzheimer DNA biobank) funded by the JPco-fuND (German Federal Ministry of Education and Research, BMBF: 01ED1619A). Full acknowledgments for the studies that contributed data can be found in the Supplementary Note. We thank the numerous participants, researchers, and staff from many studies who collected and contributed to the data. We thank the International Genomics of Alzheimer's Project (IGAP) for providing summary results data for these analyses. The investigators within IGAP contributed to the design and implementation of IGAP and/or provided data but did not participate in analysis or writing of this report. IGAP was made possible by the generous participation of the control subjects, the patients, and their families. The i-Select chips was funded by the French National Foundation on AD and related disorders. EADI was supported by the LABEX (laboratory of excellence program investment for the future) DISTALZ grant, Inserm, Institut Pasteur de Lille, Université de Lille 2 and the Lille University Hospital. GERAD was supported by the Medical Research Council (Grant $n^{\circ}$ 503480), Alzheimer's Research UK (Grant $n^{\circ}$ 503176), the Wellcome Trust (Grant $n^{\circ} 082604 / 2 / 07 / Z$ ) and German Federal Ministry of Education and Research (BMBF): Competence Network Dementia (CND) grant $n^{\circ} 01 \mathrm{GI0102}$, 01GI0711, 01GI0420. CHARGE was partly supported by the NIA/NHLBI grants AG049505, AG058589, HL105756 and AGES contract N01-AG-12100, the Icelandic Heart Association, and the Erasmus Medical Center and Erasmus University. ADGC was supported by the NIH/NIA grants: U01 AG032984, U24 AG021886, U01 AG016976, and the Alzheimer's Association grant ADGC-10-196728. This research has been conducted using the UK Biobank public resource obtained through the University of Edinburg Data Share (https://datashare.is.ed.ac.uk/handle/10283/3364).

\section{Author contributions}

A.Ru and S.v.d.L. designed and conceptualized the study, interpreted the data and drafted the paper. I.d.R. contributed to data acquisition, the analysis, interpreted the data, and co-wrote the paper. S.M.G. and N.T. contributed to the analysis and interpreted the data. S.J.v.d.L. and I.d.R. performed polygenic score analyses. L.C.C. and J.C. conducted the functional analysis of APP. H.Ho, W.v.d.F., S.J.v.d.L., and A.Ru supervised the study. All authors critically revised the paper for important intellectual content and approved the final paper. GR@ACE/DEGESCO: Study design or conception: M.Me, J.C., and A.Ru. Data generation: L.M., L.C.C., A.G.P., M.E.S., S.M.G., I.d.R., I.Q., and A.C. Sample contribution: M.Bo, J.M.G.A., M.Me, M.Ma, M.C., T.S., S.G.M., G.G.R., A.L.M., J.M., L. M.R., G.P.R., M.M.G., C.M.R., I.R.A., V.A.l., C.L., E.R.R., P.S.J., D.A.l., P.P., M.D.F., I.A.l., J.P.T., A.C., L.Ta, A.M.M., M.J.B., A.F.G., I.Q., I.H., L.M., P.G.G., E.A.M., S.V., O.S.G., A Bena, A.P.C., A.E., A.Sa, C.Ab, G.O., M.R.R., M.A.l., N.R., S.G., A.O., A.Rab, A.Bel, F.Mo, M.Z., A.C.G., J.A.P., M.F.F., E.F.M., D.B.R., M.B.S.A., P.M., R.H.V., A.A.P., A.A., L.M.P., R.S.V., E.Ge, A.L., R.B., J.F., J.L.R., S.Men, M.Ba, I.d.R., and S.M.G. Analysis: S.M.G. and I.d.R. Study supervision/management: A.C., L.Ta, M.Bo, M.Me, J.C., and A.Ru. IGAP. Critical revision: A.C.N., B.W.K., L.A.F., J.L.H., L.S.W., M.A.P.V., R.May, M.A.I., J.C.B., A.L.D.S., C.L.S., E.B., M.F., Q.Y., X.J., R.S., C.H., K.M., S.Mea, V.E.P., A.Meg, P.A.H., R. Mar, P.A., G.Sc, J.Will, and S.Se. EADB: Sample contribution. N.T., I.E.J., N.St, K.A.M., C.D., G.N., G.C., G.Sp, K.S., M.I., M.K., R.F.S., J.C.L., A.Ra, D.G., J.S.V., D.R., E.Gr, H.Ha, I.G., J.K., L.Fa, L.Fr, A.M.H., J.V., L.H., G.H., N.Sca, M.H.K., M.Y., H.Ho, W.M.v.d..F, M H.u.l., N.M.v.S., A.T.H., B.G.N., C.V.B., E.S., R.V., S.E., T.N., F.K., J.V.D., V.G., A.U., A Benu, A.K.S., B.B., C.Mas, C.F., E.C., F.Ma, G.B., I.Ap, J.Q.T., L.Ki, L.K.l., L.P., L.Tr, L.B., M.L., M.Ar, R.G., S.F., F.J., J.D.S., O.G., T.G., M.J.H., T.P., K.Bu, M.E.si, S.R.H., E.Dur, A. Ru, I.H., S.M.G., I.d.R., Y.A.L..P, A.d.M., C.C.l., J.P., S.J.v.d.L., C.G., N.B., O.H., P.B., A. H., T.K., M.E.w., O.A.S., R.N.K., J.Wilf, P.F., P.R., P.Sc, P.Sa, N.Sch, D.W., E.R., G.R., H. S., I.R., A.Sc, A.Sp, A.Sq, C.Cha, C.Chi, C.P., A.P., B.A., B.N., C.M.F., D.S., E.Da, E.düz, E. F., F.T., F.P., F.S.G., G.Gi, G.Gra, G.P., H.B., J.H., J.L., M.C.D., M.T., M.T.H., M.Schm, M. W., M.S., O.Q., O.L., P.C., P.D., P.M., R.C., S.So, S.He, S.A., S.B., S.C., T.L., V.B., V.D., P. G.K., M.M.N., M.C.D.N., O.P., W.M., A.W.L., I.Ap, C.B.F., A.A.L.K., G.B., M.Sca, M.Sp, M.V., M.Hi, K.F., L.W., M.D., P.H., and A.Ra. Analysis: N.J.A., R.M.T., V.An, N.T., I.E.J., N.St, S.J.v.d.L., I.d.R., S.M.G., B.G.B., and C.B. Studies supervision/management: S.H.H., K.A.M., C.D., G.N., G.C., G.S., K.S., M.I., M.K., R.F.S., D.G., J.S.V., D.R., E.Gr, H.Ha, I.G., J.K., L.Fr, A.M.H., J.V., L.H., G.H., N.Sca, M.H.K., M.Y., H.Ho, W.M.v.d.F., M.Hui, N.M v.S., N.J.A., J.D., M.Sche, A.K.S., C.G., N.B., O.H., P.B., A.H., T.K., J.Wilf, P.F., P.R., P.Sa P.Sc, M.Hul, N.T., I.E.J., A.T.H., B.G.N., C.V.B., E.S., R.V., S.E., P.A., A.Ru, and J.C.L. PGC-ALZ: Sample contribution. I.E.J., A.Ro, I.Sa, D.Aa, G.Se, S.B.S., S.D., D.P., S.H., I.K K., N.L.P., C.A.R., and O.A.A. Analysis: S.Ha, I.K.K., and I.E.J. Study supervision/management: O.A.A., C.M.v.D., and D.P. AD and GBCS: Sample contribution. H.Z., S.K., I.S., and K.B. Analysis: N.M.S. and A.Z. Study supervision/management: I.S.k., A.Z., and K.B.I NxC: Sample contribution. A.C.A., M.T.M., M.S.R., and C.An.

\section{Competing interests}

The authors declare no competing interests. 


\section{Additional information}

Supplementary information The online version contains supplementary material available at https://doi.org/10.1038/s41467-021-22491-8.

Correspondence and requests for materials should be addressed to S.J.v.d.L. or A.R.

Peer review information Nature Communications thanks the anonymous reviewers for their contribution to the peer review of this work. Peer reviewer reports are available.

Reprints and permission information is available at http://www.nature.com/reprints

Publisher's note Springer Nature remains neutral with regard to jurisdictional claims in published maps and institutional affiliations. (c) (i) Open Access This article is licensed under a Creative Commons Attribution 4.0 International License, which permits use, sharing, adaptation, distribution and reproduction in any medium or format, as long as you give appropriate credit to the original author(s) and the source, provide a link to the Creative Commons license, and indicate if changes were made. The images or other third party material in this article are included in the article's Creative Commons license, unless indicated otherwise in a credit line to the material. If material is not included in the article's Creative Commons license and your intended use is not permitted by statutory regulation or exceeds the permitted use, you will need to obtain permission directly from the copyright holder. To view a copy of this license, visit http://creativecommons.org/licenses/by/4.0/.

(c) The Author(s) 2021, corrected publication 2023

Itziar de Rojas (10) 1,2,306, Sonia Moreno-Grau1,2,306, Niccolo Tesi3,4,5,306, Benjamin Grenier-Boley6,306, Victor Andrade $7,8,306$, Iris E. Jansen 3,9,306, Nancy L. Pedersen ${ }^{10}$, Najada Stringa (1) ${ }^{11}$, Anna Zettergren (1) ${ }^{12}$, Isabel Hernández ${ }^{1,2}$, Laura Montrreal ${ }^{1}$, Carmen Antúnez ${ }^{13}$, Anna Antonell ${ }^{14}$, Rick M. Tankard (15) ${ }^{15}$ Joshua C. Bis ${ }^{16}$, Rebecca Sims (10 ${ }^{17}$, Céline Bellenguez (1) ${ }^{6}$, Inés Quintela ${ }^{18}$, Antonio González-Perez ${ }^{19}$, Miguel Calero (10) 20,21, Emilio Franco-Macías ${ }^{22}$, Juan Macías ${ }^{23}$, Rafael Blesa2,24, Laura Cervera-Carles (10) 2,24, Manuel Menéndez-González (10) 25,26,27, Ana Frank-García2,28,29,30, Jose Luís Royo31, Fermin Moreno²,32,33, Raquel Huerto Vilas 34,35 , Miquel Baquero ${ }^{36}$, Mónica Diez-Fairen (10) 37,38, Carmen Lage (1) 2,39, Sebastián García-Madrona 40, Pablo García-González (10) 1, Emilio Alarcón-Martín,31, Sergi Valero 1,2, Oscar Sotolongo-Grau', Abbe Ullgren ${ }^{41,42}$, Adam C. Naj ${ }^{43,44}$, Afina W. Lemstra ${ }^{3}$, Alba Benaque Alba Pérez-Cordón (10) ${ }^{1}$, Alberto Benussi (D) ${ }^{45}$, Alberto Rábano 2,21,46, Alessandro Padovani ${ }^{45}$, Alessio Squassina (10 47, Alexandre de Mendonça ${ }^{48}$, Alfonso Arias Pastor ${ }^{34,35}$, Almar A. L. Kok ${ }^{11,49}$, Alun Meggy ${ }^{50}$, Ana Belén Pastor (10) 21,46, Ana Espinosa1,2, Anaïs Corma-Gómez ${ }^{23}$, Angel Martín Montes (1) 2,29,51, Ángela Sanabria1,2, Anita L. DeStefano 52,53, Anja Schneider ${ }^{8,54}$, Annakaisa Haapasalo (1) ${ }^{55}$,

Anne Kinhult Ståhlbom 41,42, Anne Tybjærg-Hansen 56,57, Annette M. Hartmann ${ }^{58}$, Annika Spottke 54,59, Arturo Corbatón-Anchuelo60,61, Arvid Rongve (1D) 62,63, Barbara Borroni ${ }^{45}$, Beatrice Arosio 64,65 , Benedetta Nacmias (10 66,67, Børge G. Nordestgaard (10 57,68, Brian W. Kunkle ${ }^{69,70}$, Camille Charbonnier ${ }^{71}$, Carla Abdelnour ${ }^{1,2}$, Carlo Masullo ${ }^{72}$, Carmen Martínez Rodríguez ${ }^{26,73}$, Carmen Muñoz-Fernandez ${ }^{74}$, Carole Dufouil 75,76 , Caroline Graff 41,42 , Catarina B. Ferreira ${ }^{77}$, Caterina Chillotti ${ }^{78}$, Chandra A. Reynolds (1) ${ }^{79}$, Chiara Fenoglio 80 , Christine Van Broeckhoven (1) ${ }^{81,82,83}$, Christopher Clark (10) ${ }^{84}$, Claudia Pisanu (1) ${ }^{47}$, Claudia L. Satizabal (1D) 52,85,86, Clive Holmes ${ }^{87}$, Dolores Buiza-Rueda 2,88 , Dag Aarsland (10) 89,90, Dan Rujescu ${ }^{58,}$

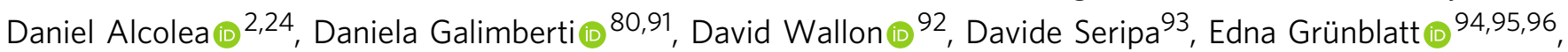
Efthimios Dardiotis 97 , Emrah Düzel ${ }^{98,99}$, Elio Scarpini ${ }^{80,91}$, Elisa Conti ${ }^{100}$, Elisa Rubino ${ }^{101}$, Ellen Gelpi ${ }^{102,103}$, Eloy Rodriguez-Rodriguez (10) 2,39 , Emmanuelle Duron 104,105,106, Eric Boerwinkle ${ }^{107,108}$, Evelyn Ferri65, Fabrizio Tagliavini ${ }^{109}$, Fahri Küçükali (10) 81,82,83, Florence Pasquier (10) 110,111, Florentino Sanchez-Garcia ${ }^{112}$, Francesca Mangialasche ${ }^{113}$, Frank Jessen ${ }^{54,114,115}$, Gaël Nicolas (10) ${ }^{73}$, Geir Selbæk (10 116,117,118, Gemma Ortega 1,2, Geneviève Chêne ${ }^{75,76}$, Georgios Hadjigeorgiou ${ }^{119}$, Giacomina Rossi ${ }^{109}$, Gianfranco Spalletta120,121, Giorgio Giaccone ${ }^{109}$, Giulia Grande ${ }^{122}$, Giuliano Binetti ${ }^{123,124}$, Goran Papenberg ${ }^{122}$, Harald Hampel ${ }^{125}$, Henri Bailly 106,126, Henrik Zetterberg (1) 127,128,129,130, Hilkka Soininen (1) 131,132, Ida K. Karlsson ${ }^{10,133,}$ Ignacio Alvarez (1) 37,38, Ildebrando Appollonio 100,134, Ina Giegling 58, Ingmar Skoog 12, Ingvild Saltvedt (135,136, Innocenzo Rainero ${ }^{137}$, Irene Rosas Allende 26,138 , Jakub Hort ${ }^{139,140}$, Janine Diehl-Schmid ${ }^{141}$, Jasper Van Dongen (10 81,82, Jean-Sebastien Vidal (106,126, Jenni Lehtisalo ${ }^{131,142}$, Jens Wiltfang 143,144,145, 
Jesper Qvist Thomassen ${ }^{56}$, Johannes Kornhuber (10) ${ }^{146}$, Jonathan L. Haines (1) 147,148, Jonathan Vogelgsang (140) ${ }^{143,149}$, Juan A. Pineda (iD ${ }^{23}$, Juan Fortea 2,24, Julius Popp 150,151,152, Jürgen Deckert ${ }^{153}$,

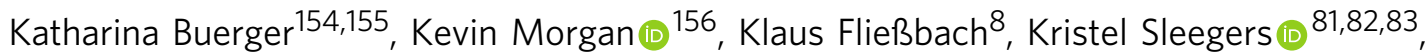
Laura Molina-Porcel ${ }^{14,102}$, Lena Kilander ${ }^{157}$, Leonie Weinhold ${ }^{158}$, Lindsay A. Farrer (159, Li-San Wang ${ }^{44}$, Luca Kleineidam 7,8, Lucia Farotti160, Lucilla Parnetti ${ }^{160}$, Lucio Tremolizzo ${ }^{100,134}$, Lucrezia Hausner ${ }^{161}$, Luisa Benussi ${ }^{124}$, Lutz Froelich ${ }^{161}$, M. Arfan Ikram (1) ${ }^{162}$, M. Candida Deniz-Naranjo ${ }^{112}$, Magda Tsolaki (iD ${ }^{163}$, Maitée Rosende-Roca ${ }^{1,2}$, Malin Löwenmark ${ }^{157}$, Marc Hulsman²,4, Marco Spallazzi ${ }^{164}$,

Margaret A. Pericak-Vance (10 ${ }^{70}$, Margaret Esiri ${ }^{165}$, María Bernal Sánchez-Arjona ${ }^{22}$, Maria Carolina Dalmasso ${ }^{7}$, María Teresa Martínez-Larrad ${ }^{60,61}$, Marina Arcaro ${ }^{91}$, Markus M. Nöthen ${ }^{166}$, Marta Fernández-Fuertes ${ }^{23}$, Martin Dichgans (10) 154,155,167, Martin Ingelsson ${ }^{157}$, Martin J. Herrmann ${ }^{153}$, Martin Scherer ${ }^{168}$, Martin Vyhnalek ${ }^{139,140}$, Mary H. Kosmidis ${ }^{169}$, Mary Yannakoulia ${ }^{170}$, Matthias Schmid 54,158 , Michael Ewers (154,155, Michael T. Heneka 8,54, Michael Wagner (10,54, Michela Scamosci ${ }^{171}$, Miia Kivipelto 113,172,173,174, Mikko Hiltunen ${ }^{175}$, Miren Zulaica2,33, Montserrat Alegret,2, Myriam Fornage ${ }^{176}$, Natalia Roberto ${ }^{1}$, Natasja M. van Schoor ${ }^{11}$, Nazib M. Seidu ${ }^{12}$, Nerisa Banaj ${ }^{120}$, Nicola J. Armstrong ${ }^{15}$, Nikolaos Scarmeas ${ }^{177,178}$, Norbert Scherbaum ${ }^{179}$, Oliver Goldhardt ${ }^{141}$, Oliver Hanon ${ }^{106,126}$, Oliver Peters ${ }^{180,181}$, Olivia Anna Skrobot ${ }^{182}$, Olivier Quenez (1) ${ }^{71}$, Ondrej Lerch ${ }^{139,140}$, Paola Bossù (10) 183, Paolo Caffarra ${ }^{184}$, Paolo Dionigi Rossi65, Paraskevi Sakka185, Patrizia Mecocci ${ }^{171}$, Per Hoffmann ${ }^{166,186}$, Peter A. Holmans (1) ${ }^{17}$, Peter Fischer ${ }^{187}$, Peter Riederer ${ }^{188}$, Qiong Yang (1) ${ }^{53}$, Rachel Marshall ${ }^{17}$, Rajesh N. Kalaria ${ }^{189,190,}$ Richard Mayeux ${ }^{191,192,193}$, Rik Vandenberghe ${ }^{194,195}$, Roberta Cecchetti ${ }^{171}$, Roberta Ghidoni ${ }^{124}$, Ruth Frikke-Schmidt56,57, Sandro Sorbi66,67, Sara Hägg (10 ${ }^{10}$, Sebastiaan Engelborghs ${ }^{196,197,198,199}$, Seppo Helisalmi ${ }^{200}$, Sigrid Botne Sando 201,202 , Silke Kern ${ }^{12}$, Silvana Archetti ${ }^{203}$, Silvia Boschi ${ }^{137}$, Silvia Fostinelli ${ }^{124}$, Silvia Gil1, Silvia Mendoza ${ }^{204}$, Simon Mead ${ }^{205}$, Simona Ciccone ${ }^{65}$, Srdjan Djurovic (D) 206,207, Stefanie Heilmann-Heimbach ${ }^{166}$, Steffi Riedel-Heller ${ }^{208}$, Teemu Kuulasmaa ${ }^{175}$, Teodoro del Ser ${ }^{209}$, Thibaud Lebouvier ${ }^{110,111}$, Thomas Polak ${ }^{153}$, Tiia Ngandu133,142, Timo Grimmer ${ }^{141}$, Valentina Bessi (i) 66,210, Valentina Escott-Price (1) 17,211, Vilmantas Giedraitis (15) ${ }^{157}$, Vincent Deramecourt ${ }^{110,111}$, Wolfgang Maier ${ }^{8,54}$, Xueqiu Jian (10 85 , Yolande A. L. Pijnenburg ${ }^{3}$, EADB contributors ${ }^{\star}$, The GR@ACE study group*, DEGESCO consortium*, IGAP (ADGC, CHARGE, EADI, GERAD) ${ }^{\star}$, PGC-ALZ consortia*, Patrick Gavin Kehoe (1) ${ }^{182}$, Guillermo Garcia-Ribas ${ }^{40}$, Pascual Sánchez-Juan (1) 2,39, Pau Pastor (1) ${ }^{37,38}$, Jordi Pérez-Tur (1) 2,212,213, Gerard Piñol-Ripol134,35, Adolfo Lopez de Munain (10) 2,32,33,214, Jose María García-Alberca (10,204, María J. Bullido (iD) 2,30,215,216, Victoria Álvarez 26,138, Alberto Lleó (iD 2,24, Luis M. Real (iD 23,217, Pablo Mir 2,88,

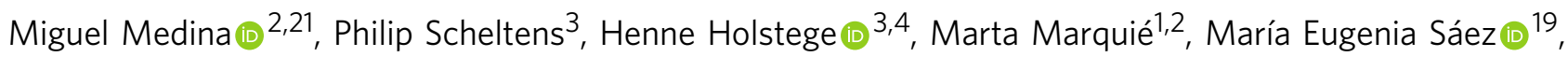

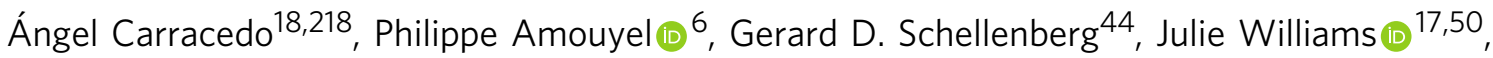
Sudha Seshadri (15) 52,85,219, Cornelia M. van Duijn (1) 162,220, Karen A. Mather (1021,222, Raquel Sánchez-Valle ${ }^{14}$, Manuel Serrano-Ríos ${ }^{60,61}$, Adelina Orellana 1,2, Lluís Tárraga ${ }^{1,2}$, Kaj Blennow (1) 127,128, Martijn Huisman ${ }^{11,223,}$ Ole A. Andreassen (1024,225, Danielle Posthuma9 , Jordi Clarimón 2,24,307, Mercè Boada (1) 1,307, Wiesje M. van der Flier (1D3,307, Alfredo Ramirez (1) 7,8,54,226,307, Jean-Charles Lambert (1D,307, Sven J. van der Lee (iD ${ }^{3,4,307 凶}$ \& Agustín Ruiz (iD) 1,2,307凶

\footnotetext{
${ }^{1}$ Research Center and Memory clinic Fundació ACE, Institut Català de Neurociències Aplicades, Universitat Internacional de Catalunya, Barcelona, Spain. ${ }^{2}$ CIBERNED, Network Center for Biomedical Research in Neurodegenerative Diseases, National Institute of Health Carlos III, Madrid, Spain. ${ }^{3}$ Alzheimer Center Amsterdam, Department of Neurology, Amsterdam Neuroscience, Vrije Universiteit Amsterdam, Amsterdam UMC,

Amsterdam, The Netherlands. ${ }^{4}$ Section Genomics of Neurodegenerative Diseases and Aging, Department of Clinical Genetics, Vrije Universiteit Amsterdam, Amsterdam UMC, Amsterdam, The Netherlands. ${ }^{5}$ Delft Bioinformatics Lab, Delft Univeristy of Technology, Delft, The Netherlands. 6Univ. Lille, Inserm, Institut Pasteur de Lille, CHU Lille, U1167-Labex DISTALZ-RID-AGE-Risk Factors and Molecular Determinants of Aging-Related Diseases, Lille, France. 'Division of Neurogenetics and Molecular Psychiatry, Department of Psychiatry and Psychotherapy, University of Cologne, Medical Faculty, Cologne, Germany. ${ }^{8}$ Department of Neurodegenerative diseases and Geriatric Psychiatry, University Clinic Bonn, Bonn, Germany. ${ }^{9}$ Department of Complex Trait Genetics, Center for Neurogenomics and Cognitive Research, Amsterdam Neuroscience, VU University,
} 
Amsterdam, The Netherlands. ${ }^{10}$ Department of Medical Epidemiology and Biostatistics, Karolinska Institutet, Stockholm, Sweden. ${ }^{11}$ Amsterdam UMC-Vrije Universiteit Amsterdam, Department of Epidemiology and Data Science, Amsterdam Public Health Research Institute, Amsterdam, The Netherlands. ${ }^{12}$ Neuropsychiatric Epidemiology Unit, Department of Psychiatry and Neurochemistry, Institute of Neuroscience and Physiology, Sahlgrenska Academy, Centre for Ageing and Health (AgeCap), University of Gothenburg, Gothenburg, Sweden. ${ }^{13}$ Unidad de Demencias, Hospital Clínico Universitario Virgen de la Arrixaca, Murcia, Spain. ${ }^{14}$ Alzheimer's disease and other cognitive disorders unit. Service of Neurology, Hospital Clínic of Barcelona. Institut d'Investigacions Biomèdiques August Pi i Sunyer, University of Barcelona, Barcelona, Spain. ${ }^{15}$ Mathematics and Statistics, Murdoch University, Perth, WA, Australia. ${ }^{16}$ Cardiovascular Health Research Unit, Department of Medicine, University of Washington, Seattle, WA, USA. ${ }^{17}$ Division of Psychological Medicine and Clinial Neurosciences, MRC Centre for Neuropsychiatric Genetics and Genomics, Cardiff University, Cardiff, UK. ${ }^{18}$ Grupo de Medicina Xenómica, Centro Nacional de Genotipado (CEGEN-PRB3-ISCIII), Universidade de Santiago de Compostela, Santiago de Compostela, Spain. ${ }^{19} \mathrm{CAEBI}$, Centro Andaluz de Estudios Bioinformáticos, Sevilla, Spain. ${ }^{20}$ UFIEC, Instituto de Salud Carlos III, Madrid, Spain. ${ }^{21}$ CIEN Foundation/Queen Sofia Foundation Alzheimer Center, Madrid, Spain. ${ }^{22}$ Unidad de Demencias, Servicio de Neurología y Neurofisiología, Instituto de Biomedicina de Sevilla (IBiS), Hospital Universitario Virgen del Rocío/CSIC/Universidad de Sevilla, Sevilla, Spain. ${ }^{23}$ Unidad Clínica de Enfermedades Infecciosas y Microbiología, Hospital Universitario de Valme, Sevilla, Spain. ${ }^{24}$ Department of Neurology, II B Sant Pau, Hospital de la Santa Creu i Sant Pau, Universitat Autònoma de Barcelona, Barcelona, Spain. ${ }^{25}$ Servicio de Neurología, Hospital Universitario Central de Asturias, Oviedo, Spain. ${ }^{26}$ Instituto de Investigación Sanitaria del Principado de Asturias (ISPA), Oviedo, Spain. ${ }^{27}$ Departamento de Medicina, Universidad de Oviedo, Oviedo, Spain. ${ }^{28}$ Department of Neurology, La Paz University Hospital, Instituto de Investigación Sanitaria del Hospital Universitario La Paz, IdiPAZ, Madrid, Spain. ${ }^{29}$ Hospital La Paz Institute for Health Research, IdiPAZ, Madrid, Spain. ${ }^{30}$ Universidad Autónoma de Madrid, Madrid, Spain. ${ }^{31}$ Departamento de Especialidades Quirúrgicas, Bioquímicas e Inmunología, School of Medicine, University of Málaga, Málaga, Spain. ${ }^{32}$ Department of Neurology, Hospital Universitario Donostia, San Sebastian, Spain. ${ }^{33}$ Neurosciences Area, Instituto Biodonostia, San Sebastian, Spain. ${ }^{34}$ Unitat Trastorns Cognitius, Hospital Universitari Santa Maria de Lleida, Lleida, Spain. ${ }^{35}$ Institut de Recerca Biomedica de Lleida (IRBLLeida), Lleida, Spain. ${ }^{36}$ Servei de Neurologia, Hospital Universitari i Politècnic La Fe, Valencia, Spain. ${ }^{37}$ Fundació Docència i Recerca MútuaTerrassa, Terrassa, Barcelona, Spain. ${ }^{38}$ Memory Disorders Unit, Department of Neurology, Hospital Universitari Mutua de Terrassa, Terrassa, Barcelona, Spain. ${ }^{39}$ Neurology Service, Marqués de Valdecilla University Hospital (University of Cantabria and IDIVAL), Santander, Spain. ${ }^{40}$ Hospital Universitario Ramon y Cajal, IRYCIS, Madrid, Spain. ${ }^{41}$ Karolinska Institutet, Center for Alzheimer Research, Department NVS, Division of Neurogeriatrics, Stockholm, Sweden. ${ }^{42}$ Unit for Hereditary Dementias, Theme Aging, Karolinska University Hospital-Solna, Stockholm, Sweden. ${ }^{43}$ Department of Biostatistics, Epidemiology and Informatics, University of Pennsylvania Perelman School of Medicine, Philadelphia, PA, USA. ${ }^{44}$ Penn Neurodegeneration Genomics Center, Department of Pathology and Laboratory Medicine, University of Pennsylvania Perelman School of Medicine, Philadelphia, PA, USA. ${ }^{45}$ Centre for Neurodegenerative Disorders, Department of Clinical and Experimental Sciences, University of Brescia, Brescia, Italy. ${ }^{46}$ BT-CIEN, Madrid, Spain. ${ }^{47}$ Department of Biomedical Sciences, Section of Neuroscience and Clinical Pharmacology, University of Cagliari, Cagliari, Italy. ${ }^{48}$ Faculty of Medicine, University of Lisbon, Lisbon, Portugal. ${ }^{49}$ Amsterdam UMC, Vrije Universiteit Amsterdam, Department of Psychiatry, Amsterdam Public Health Research Institute, Amsterdam, The Netherlands. ${ }^{50}$ UK Dementia Research Institute at Cardiff, Cardiff University, Cardiff, UK. ${ }^{51}$ Department of Neurology, La Paz University Hospital, Madrid, Spain. ${ }^{52}$ Department of Neurology, Boston University School of Medicine, Boston, MA, USA. ${ }^{53}$ Department of Biostatistics, Boston University School of Public Health, Boston, MA, USA. ${ }^{54}$ German Center for Neurodegenerative Diseases (DZNE), Bonn, Germany. ${ }^{55}$ A.I Virtanen Institute for Molecular Sciences, University of Eastern Finland, Kuopio, Finland. ${ }^{56}$ Department of Clinical Biochemistry, Rigshospitalet, Copenhagen, Denmark. ${ }^{57}$ Department of Clinical Medicine, Faculty of Health and Medical Sciences, University of Copenhagen, Copenhagen, Denmark. ${ }^{58}$ Martin-Luther-University Halle-Wittenberg, University Clinic and Outpatient Clinic for Psychiatry, Psychotherapy and Psychosomatics, Halle (Saale), Germany. ${ }^{59}$ Department of Neurology, University of Bonn, Bonn, Germany. ${ }^{60}$ Instituto de Investigación Sanitaria, Hospital Clínico San Carlos (IdISSC), Madrid, Spain. ${ }^{61}$ Spanish Biomedical Research Centre in Diabetes and Associated Metabolic Disorders (CIBERDEM), Madrid, Spain. ${ }^{62}$ Haugesund Hospital, Helse Fonna, Department of Research and Innovation, Haugesund, Norway. ${ }^{63}$ University of Bergen, Institute of Clinical Medicine (K1), Bergen, Norway. ${ }^{64}$ Department of Clinical Sciences and Community Health, University of Milan, Milan, Italy. ${ }^{65}$ Geriatic Unit, Fondazione Cà Granda, IRCCS Ospedale Maggiore Policlinico, Milan, Italy. ${ }^{66}$ Department of Neuroscience, Psychology, Drug Research and Child Health University of Florence, Florence, Italy. ${ }^{67}$ IRCCS Fondazione Don Carlo Gnocchi, Florence, Italy. ${ }^{68}$ Department of Clinical Biochemistry, Herlev Gentofte Hospital, Herlev, Denmark. ${ }^{69}$ Dr. John T. Macdonald Foundation Department of Human Genetics, University of Miami Miller School of Medicine, Miami, FL, USA. ${ }^{70}$ John P. Hussman Institute for Human Genomics, University of Miami Miller School of Medicine, Miami, FL, USA. ${ }^{71}$ Normandie Univ, UNIROUEN, Inserm U1245, CHU Rouen, Department of Genetics and CNR-MAJ, FHU G4 Génomique, F-76000 Rouen, France. ${ }^{72}$ Institute of Neurology, Catholic University of the Sacred Heart, School of Medicine, Milan, Italy. ${ }^{73}$ Hospital de Cabueñes, Gijón, Spain. ${ }^{74}$ Servicio de Neurología, Hospital Universitario de Gran Canaria Dr.Negrín, Las Palmas, Spain. ${ }^{75}$ Inserm, Bordeaux Population Health Research Center, UMR 1219, Univ. Bordeaux, ISPED, CIC 1401-EC, Univ Bordeaux, Bordeaux, France. ${ }^{76} \mathrm{CHU}$ de Bordeaux, Pole de Santé Publique, Bordeaux, France. ${ }^{77}$ Instituto de Medicina Molecular João lobo Antunes, Faculdade de Medicina, Universidade de Lisboa, Lisboa, Portugal. ${ }^{78}$ Unit of Clinical Pharmacology, University Hospital of Cagliari, Cagliari, Italy. ${ }^{79}$ Department of Psychology, University of California-Riverside, Riverside, CA, USA. ${ }^{80}$ University of Milan, Dino Ferrari Center, Milan, Italy. ${ }^{81}$ VIB Center for Molecular Neurology, Antwerp, Belgium. ${ }^{82}$ Laboratory of Neurogenetics, Institute Born-Bunge, Antwerp, Belgium. ${ }^{83}$ Department of Biomedical Sciences, University of Antwerp., Antwerp, Belgium. ${ }^{84}$ Insititute for Regenerative Medicine, University of Zürich, Zürich, Switzerland. ${ }^{85} \mathrm{Glenn}$ Biggs Institute for Alzheimer's and Neurodegenerative Diseases, San Antonio, TX, USA. ${ }^{86}$ Department of Population Health Sciences, UT Health San Antonio, San Antonio, TX, USA. ${ }^{87}$ Division of Clinical Neurosciences, School of Medicine, University of Southampton, Southampton, UK. ${ }^{88}$ Unidad de Trastornos del Movimiento, Servicio de Neurología y Neurofisiología, Instituto de Biomedicina de Sevilla (IBiS), Hospital Universitario Virgen del Rocío/CSIC/Universidad de Sevilla, Sevilla, Spain. ${ }^{89}$ Department of Old Age Psychiatry, Institute of Psychiatry, Psychology \& Neuroscience, King's College London, London, UK. ${ }^{0} \mathrm{Centre}$ of AgeRelated Medicine, Stavanger University Hospital, Stavanger, Norway. ${ }^{91}$ Fondazione IRCCS Ca' Granda, Ospedale Policlinico, Milan, Italy. ${ }^{92}$ Normandie Univ, UNIROUEN, Inserm U1245, CHU Rouen, Department of Neurology and CNR-MAJ, FHU G4 Génomique, F-76000 Rouen, France. ${ }^{93}$ Complex Structure of Geriatrics, Department of Medical Sciences Fondazione IRCCS Casa Sollievo della Sofferenza, San Giovanni Rotondo (FG), Italy. ${ }^{94}$ Department of Child and Adolescent Psychiatry and Psychotherapy, Psychiatric University Hospital Zurich (PUK), University of Zurich, Zurich, Switzerland. ${ }^{95}$ Neuroscience Center Zurich, University of Zurich and ETH Zurich, Zurich, Switzerland. ${ }^{96}$ Zurich Center for Integrative Human Physiology, University of Zurich, Zurich, Switzerland. ${ }^{97}$ School of Medicine, University of Thessaly, Larissa, Greece. ${ }^{98}$ German Center for Neurodegenerative Diseases (DZNE), Magdeburg, Germany. ${ }^{99}$ Institute of Cognitive Neurology and Dementia Research (IKND), Otto-von-Guericke University, Magdeburg, Germany. ${ }^{100}$ School of Medicine and Surgery, University of Milano-Bicocca and Milan Center for Neuroscience, Milan, Italy. ${ }^{101}$ Department of Neuroscience and Mental Health, AOU Città della Salute e della Scienza di Torino, Torino, Italy. ${ }^{102}$ Neurological Tissue Bank of the Biobanc-Hospital Clinic-IDIBAPS, Institut d'Investigacions Biomèdiques August Pi i Sunyer, Barcelona, Spain. ${ }^{103}$ Division of Neuropathology and Neurochemistry, Department of Neurology, Medical University of Vienna, Vienna, Austria. ${ }^{104}$ APHP, Hôpital 
Brousse, equipe INSERM 1178, MOODS, Villejuif, France. ${ }^{105}$ Université Paris-Saclay, UVSQ, Inserm, CESP, Team MOODS, Le Kremlin-Bicêtre, Paris, France. ${ }^{106} \mathrm{APHP}$, Hôpital Broca, Paris, France. ${ }^{107} \mathrm{~S} c h o o l$ of Public Health, Human Genetics Center, University of Texas Health Science Center at Houston, Houston, TX, USA. ${ }^{108}$ Human Genome Sequencing Center, Baylor College of Medicine, Houston, TX, USA. ${ }^{109}$ Fondazione IRCCS Istituto Neurologico Carlo Besta, Milan, Italy. ${ }^{110}$ Inserm U1172, CHU, DISTAlz, LiCEND, Univ Lille, Lille, France. ${ }^{111} \mathrm{CHU}$ CNR-MAJ, Lille, France. ${ }^{112}$ Servicio de Inmunología, Hospital Universitario de Gran Canaria Dr. Negrín, Las Palmas de Gran Canaria, Spain. ${ }^{113}$ Division of Clinical Geriatrics, Center for Alzheimer Research, Department of Neurobiology, Care Sciences and Society (NVS), Karolinska Institutet, Stockholm, Sweden. ${ }^{114}$ Department of Psychiatry and Psychotherapy, University of Cologne, Medical Faculty, Cologne, Germany. ${ }^{115}$ Excellence Cluster on Cellular Stress Responses in Aging-Associated Diseases (CECAD), University of Cologne, Cologne, Germany. ${ }^{116}$ Norwegian National Advisory Unit on Ageing and Health, Vestfold Hospital Trust, Tønsberg, Norway. ${ }^{117}$ Department of Geriatric Medicine, Oslo University Hospital, Oslo, Norway. ${ }^{118}$ Institute of Clinical Medicine, University of Oslo, Oslo, Norway. ${ }^{119}$ Department of Neurology, Medical School, University of Cyprus, Nicosia, Cyprus. ${ }^{120}$ Laboratory of Neuropsychiatry, IRCCS Santa Lucia Foundation, Rome, Italy. ${ }^{121}$ Beth K. and Stuart C. Yudofsky Division of Neuropsychiatry, Department of Psychiatry and Behavioral Sciences, Baylor College of Medicine, Houston, TX, USA. ${ }^{122}$ Aging Research Center, Department of Neurobiology, Care Sciences and Society, Karolinska Institutet and Stockholm University, Stockholm, Sweden. ${ }^{123}$ MAC-Memory Clinic, IRCCS Istituto Centro San Giovanni di Dio Fatebenefratelli, Brescia, Italy. ${ }^{124}$ Molecular Markers Laboratory, IRCCS Istituto Centro San Giovanni di Dio Fatebenefratelli, Brescia, Italy. ${ }^{125}$ Sorbonne University, GRC n 21 , Alzheimer Precision Medicine (APM), AP-HP, Pitié-Salpêtrière Hospital, Paris, France. ${ }^{126}$ EA 4468, Sorbonne Paris Cité, Université Paris Descartes, Paris, France. ${ }^{127}$ Clinical Neurochemistry Laboratory, Sahlgrenska University Hospital, Mölndal, Sweden. ${ }^{128}$ Department of Psychiatry and Neurochemistry, Institute of Neuroscience and Physiology, Sahlgrenska Academy at the University of Gothenburg, Gothenburg, Sweden. ${ }^{129}$ Department of Neurodegenerative Disease, UCL Institute of Neurology, London, UK. ${ }^{130}$ UK Dementia Research Institute at UCL, London, UK. ${ }^{131}$ Institute of Clinical Medicine Neurology, University of Eastern Finland, Kuopio, Finland. ${ }^{132}$ Neurocenter, neurology, Kuopio University Hospital, Kuopio, Finland. ${ }^{133}$ Institute for Gerontology and Aging Research Network-Jönköping (ARN-J), School of Health and Welfare, Jönköping University, Jönköping, Sweden. ${ }^{134}$ Neurology Unit, 'San Gerardo' hospital, Monza, Italy. ${ }^{135}$ Department of Geriatrics, Clinic of Medicine, St Olavs Hospital, University Hospital of Trondheim, Trondheim, Norway. ${ }^{136}$ Department of Neuromedicine and Movement Science, Norwegian University of Science and Technhology (NTNU), Trondheim, Norway. ${ }^{137}$ Department of Neuroscience "Rita Levi Montalcini", University of Torino, Torino, Italy. ${ }^{138}$ Laboratorio de Genética, Hospital Universitario Central de Asturias, Oviedo, Spain. ${ }^{139}$ Memory Clinic, Department of Neurology, 2nd Faculty of Medicine and Motol University Hospital, Charles University, Prague, Czech Republic. ${ }^{140}$ International Clinical Research Center, St. Anne's University Hospital Brno, Brno, Czech Republic. ${ }^{141}$ Department of Psychiatry and Psychotherapy, School of Medicine Klinikum rechts der Isar, Technical University of Munich, Munich, Germany. ${ }^{142}$ Population Health Unit, Finnish Institute for Health and Welfare, Helsinki, Finland. ${ }^{143}$ Department of Psychiatry and Psychotherapy, University Medical Center Goettingen, Goettingen, Germany. ${ }^{144}$ German Center for Neurodegenerative Diseases (DZNE), Goettingen, Germany. ${ }^{145}$ Neurosciences and Signaling Group, Institute of Biomedicine (iBiMED), Department of Medical Sciences, University of Aveiro, Aveiro, Portugal. ${ }^{146}$ Department of Psychiatry and Psychotherapy, Universitätsklinikum Erlangen, Friedrich-Alexander Universität Erlangen-Nürnberg, Erlangen, Germany. ${ }^{147}$ Department of Population \& Quantitative Health Sciences, Case Western Reserve University, Cleveland, OH, USA. ${ }^{148}$ Cleveland Institute for Computational Biology, Case Western Reserve University, Cleveland, OH, USA. ${ }^{149}$ Translational Neuroscience Laboratory, McLean Hospital, Harvard Medical School, Belmont, MA, USA. ${ }^{150}$ Department of Geriatric Psychiatry, University Hospital of Psychiatry Zürich, Zürich, Switzerland. ${ }^{151}$ University of Zürich, Zürich, Switzerland. ${ }^{152}$ Old age Psychiatry, University Hospital of Lausanne, Lausanne, Switzerland. ${ }^{153}$ Department of Psychiatry, Psychosomatics and Psychotherapy, Center of Mental Health, University Hospital, Wuerzburg, Germany. ${ }^{154}$ Institute for Stroke and Dementia Research, Klinikum der Universität München, Ludwig-Maximilians-Universität LMU, Munich, Germany. ${ }^{155}$ German Center for Neurodegenerative Diseases (DZNE), Munich, Germany. ${ }^{156}$ Schools of Life Sciences and Medicine, University of Nottingham, Nottingham, UK. ${ }^{157}$ Department of Public Health and Caring Sciences/Geriatrics, Uppsala, Sweden. ${ }^{158}$ Institute of Medical Biometry, Informatics and Epidemiology, University Hospital of Bonn, Bonn, Germany. ${ }^{159}$ Departments of Medicine (Biomedical Genetics), Neurology, Ophthalmology, Epidemiology, and Biostatistics, Boston University Schools of Medicine and Public Health, Boston, MA, USA. ${ }^{160}$ Centre for Memory Disturbances, Lab of Clinical Neurochemistry, Section of Neurology, University of Perugia, Perugia, Italy. ${ }^{161}$ Department of Geriatric Psychiatry, Central Institute for Mental Health Mannheim, Medical Faculty Mannheim, University of Heidelberg, Heidelberg, Germany. ${ }^{162}$ Department of Epidemiology, Erasmus Medical Center, Rotterdam, The Netherlands. ${ }^{163} 1$ st Department of Neurology Aristotle University of Thessaloniki, Thessaloniki, Greece. ${ }^{164}$ Azienda Ospedaliero-Universitaria, Parma, Italy. ${ }^{165}$ Nuffield Department of Clinical Neurosciences, Oxford, UK. ${ }^{166}$ Institute of Human Genetics, University of Bonn, School of Medicine \& University Hospital Bonn, Bonn, Germany. ${ }^{167}$ Munich Cluster for Systems Neurology (SyNergy), Munich, Germany. ${ }^{168}$ Department of Primary Medical Care, University Medical Centre Hamburg-Eppendorf, Hamburg, Germany. ${ }^{169}$ Laboratory of Cognitive Neuroscience, School of Psychology, Aristotle University of Thessaloniki, Thessaloniki, Greece. ${ }^{170}$ Department of Nutrition and Dietetics, Harokopio University, Athens, Greece. ${ }^{171}$ Institute of Gerontology and Geriatrics, Department of Medicine, University of Perugia, Perugia, Italy. ${ }^{172}$ Institute of Public Health and Clinical Nutrition, University of Eastern Finland, Kuopio, Finland. ${ }^{173}$ Neuroepidemiology and Ageing Research Unit, School of Public Health, Imperial College London, London, UK. ${ }^{174}$ Stockholms Sjukhem, Research \& Development Unit, Stockholm, Sweden. ${ }^{175}$ Institute of Biomedicine, University of Eastern Finland, Kuopio, Finland. ${ }^{176}$ Brown Foundation Institute of Molecular Medicine, University of Texas Health Sciences Center at Houston, Houston, TX, USA. ${ }^{177}$ 1st Department of Neurology, Aiginition Hospital, National and Kapodistrian University of Athens, Medical School, Athens, Greece. ${ }^{178}$ Taub Institute for Research in Alzheimer's Disease and the Aging Brain, The Gertrude H. Sergievsky Center, Depatment of Neurology, Columbia University, New York, NY, USA. ${ }^{179}$ LVR-Hospital Essen, Department of Psychiatry and Psychotherapy, Medical Faculty, University of Duisburg-Essen, Essen, Germany. ${ }^{180}$ Department of Psychiatry and Psychotherapy and Experimental and Clinical Research Center (ECRC), Charité-Universitätsmedizin Berlin, Berlin, Germany. ${ }^{181}$ German Center for Neurodegenerative Diseases (DZNE), Berlin, Germany. ${ }^{182}$ Bristol Medical School (THS), University of Bristol, Southmead Hospital, Bristol, UK. ${ }^{183}$ Experimental Neuro-psychobiology Laboratory, Department of Clinical and Behavioral Neurology, IRCCS Santa Lucia Foundation, Rome, Italy. ${ }^{184}$ Unit of Neuroscience, DIMEC, University of Parma, Parma, Italy. ${ }^{185}$ Athens Association of Alzheimer's disease and Related Disorders, Athens, Greece. ${ }^{186}$ Institute of Medical Genetics and Pathology, University Hospital Basel, Basel, Switzerland. ${ }^{187}$ Department of Psychiatry, Social Medicine Center East- Donauspital, Vienna, Austria. ${ }^{188}$ Center of Mental Health, Clinic and Policlinic of Psychiatry, Psychosomatics and Psychotherapy, University Hospital of Würzburg, Würzburg, Germany.

${ }^{189}$ Translational and Clincial Research Institute, Newcastle University, Newcastle upon Tyne, UK. ${ }^{190}$ Campus for Ageing anf Vitality, Newcastle upon Tyne, UK. ${ }^{191}$ Taub Institute on Alzheimer's Disease and the Aging Brain, Department of Neurology, Columbia University, New York, NY, USA. ${ }^{192}$ Gertrude H. Sergievsky Center, Columbia University, New York, NY, USA. ${ }^{193}$ Department of Neurology, Columbia University, New York, NY, USA. ${ }^{194}$ Laboratory for Cognitive Neurology, Department of Neurosciences, University of Leuven, Leuven, Belgium. ${ }^{195}$ Neurology Department, University Hospitals Leuven, Leuven, Belgium. ${ }^{196}$ Center for Neurosciences, Vrije Universiteit Brussel (VUB), Brussels, Belgium. ${ }^{197}$ Reference Center for Biological Markers of Dementia (BIODEM), University of Antwerp, Antwerp, Belgium. ${ }^{198}$ Institute Born-Bunge, University of Antwerp, Antwerp, Belgium. ${ }^{199}$ Department of Neurology, VUB University Hospital Brussels (UZ Brussel), Brussels, Belgium. ${ }^{200}$ Institute of Clinical Medicine, Internal 
Medicine, University of Eastern Finland, Kuopio, Finland. ${ }^{201}$ Department of Neurology and Clinical Neurophysiology, University Hospital of Trondheim, Trondheim, Norway. ${ }^{202}$ Department of Neuromedicine and Movement Science, Faculty of Medicine and Health Sciences, Norwegian University of Science and Technology, Trondheim, Norway. ${ }^{203}$ Department of Laboratory Diagnostics, III Laboratory of Analysis, Brescia Hospital, Brescia, Italy. ${ }^{204}$ Alzheimer Research Center \& Memory Clinic, Andalusian Institute for Neuroscience, Málaga, Spain. ${ }^{205}$ MRC Prion Unit at UCL, Institute of Prion Diseases, London, UK. ${ }^{206}$ Department of Medical Genetics, Oslo University Hospital, Oslo, Norway. ${ }^{207}$ NORMENT, Department of Clinical Science, University of Bergen, Bergen, Norway. ${ }^{208}$ Institute of Social Medicine, Occupational Health and Public Health, University of Leipzig, Leipzig, Germany. ${ }^{209}$ Department of Neurology/CIEN Foundation/Queen Sofia Foundation Alzheimer Center, Madrid, Spain. ${ }^{210}$ Azienda Ospedaliero-Universitaria Careggi Largo Brambilla, Florence, Italy. ${ }^{211}$ UKDRI Cardiff, Cardiff University, Cardiff, UK. ${ }^{212}$ Unitat de Genètica Molecular, Institut de Biomedicina de València-CSIC, Valencia, Spain. ${ }^{213}$ Unidad Mixta de Neurologia Genètica, Instituto de Investigación Sanitaria La Fe, Valencia, Spain. ${ }^{214}$ Department of Neurosciences, Faculty of Medicine and Nursery, University of the Basque Country, San Sebastián, Spain. ${ }^{215}$ Centro de Biología Molecular Severo Ochoa (UAM-CSIC), Madrid, Spain. ${ }^{216}$ Instituto de Investigacion Sanitaria 'Hospital la Paz' (IdIPaz), Madrid, Spain. ${ }^{217}$ Departamento de Especialidades Quirúrgicas, Bioquímica e Inmunología. Facultad de Medicina, Universidad de Málaga, Málaga, Spain. ${ }^{218}$ Fundación Pública Galega de Medicina Xenómica-CIBERER-IDIS, Santiago de Compostela, Spain. ${ }^{219}$ Framingham Heart Study, Framingham, MA, USA. ${ }^{220}$ Nuffield Department of Population Health, University of Oxford, Oxford, UK. ${ }^{221}$ Centre for Healthy Brain Ageing (CHeBA), School of Psychiatry, Faculty of Medicine, University of New South Wales, Sydney, NSW, Australia. ${ }^{222}$ Neuroscience Research Australia, Sydney, NSW, Australia. ${ }^{223}$ Department of Sociology, VU University, Amsterdam, The Netherlands. ${ }^{224}$ NORMENT Centre, Institute of Clinical Medicine, University of Oslo, Oslo, Norway. ${ }^{225}$ Division of Mental Health and Addiction, Oslo University Hospital, Oslo, Norway. ${ }^{226}$ Department of Psychiatry, Glenn Biggs Institute for Alzheimer's and Neurodegenerative Diseases, San Antonio, TX, USA. ${ }^{306}$ These authors contributed equally: Itziar de Rojas, Sonia Moreno-Grau, Niccolo Tesi, Benjamin Grenier-Boley, Victor Andrade, Iris E. Jansen. ${ }^{307}$ These authors jointly supervised this work: Jordi Clarimón, Mercè Boada, Wiesje M. van der Flier, Alfredo Ramirez, Jean-Charles Lambert, Sven J. van der Lee, Agustín Ruiz. *Lists of

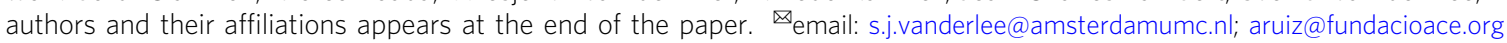

\section{EADB contributors}

A. David Smith 227,228, Aldo Saenz ${ }^{229}$, Alessandra Bizzarro ${ }^{230}$, Alessandra Lauria ${ }^{230}$, Alessandro Vacca ${ }^{137}$, Alina Solomon 113,131, Anna Anastasiou 163, Anna Richardson231,232, Anne Boland ${ }^{233}$, Anne Koivisto 234,235, Antonio Daniele ${ }^{236}$, Antonio Greco ${ }^{237}$, Arnaoutoglou Marianthi ${ }^{163}$, Bernadette McGuinness ${ }^{238}$, Bertrand Fin ${ }^{233}$, Camilla Ferrari66, Carlo Custodero 239 , Carlo Ferrarese 100,134, Carlos Ingino 240,241, Carlos Mangone 240,242, Carlos Reyes Toso 240 , Carmen Martínez ${ }^{26,243}$, Carolina Cuesta 244,245 , Carolina Muchnik 240 , Catharine Joachim ${ }^{246}$, Cecilia Ortiz ${ }^{247}$, Céline Besse 233 , Charlotte Johansson ${ }^{41,42}$, Chiara Paola Zoia100, Christoph Laske248,249, Costas Anastasiou170, Dana Lis Palacio250,251, Daniel G. Politis244,245,252, Daniel Janowitz 253 , David Craig 238 , David M. Mann ${ }^{254}$, David Neary ${ }^{231}$, Deckert Jürgen ${ }^{153}$, Delphine Daian ${ }^{233}$, Diyana Belezhanska ${ }^{255}$, Eduardo Kohler ${ }^{256}$, Eduardo M. Castaño ${ }^{257,258}$, Effrosyni Koutsouraki ${ }^{163}$, Elena Chipi ${ }^{160}$, Ellen De Roeck ${ }^{196}$, Emanuele Costantini ${ }^{236}$, Emma R. L. C. Vardy ${ }^{259}$, Fabrizio Piras ${ }^{120}$, Fausto Roveta ${ }^{137}$, Federica Piras ${ }^{120}$, Federico Ariel Prestia 245,257 , Francesca Assogna ${ }^{120}$, Francesca Salani ${ }^{183}$, Gessica Sala ${ }^{100}$, Giordano Lacidogna 236 , Gisela Novack 257,258 , Gordon Wilcock ${ }^{260}$, Håkan Thonberg41,42, Heike Kölsch²61, Heike Weber ${ }^{153}$, Henning Boecker ${ }^{54,262}$, Ignacio Etchepareborda250,251, Irene Piaceri66, Jaakko Tuomilehto ${ }^{142,263,264,265}$, Jaana Lindström ${ }^{142}$, Jan Laczo ${ }^{139,140}$, Janet Johnston ${ }^{238}$, Jean-François Deleuze ${ }^{233}$, Jenny Harris ${ }^{231}$, Jonathan M. Schott 266 , Josef Priller ${ }^{181,267}$, Juan Ignacio Bacha247, Julie Snowden231,232, Julieta Lisso ${ }^{268}$, Kalina Yonkova Mihova ${ }^{269}$, Latchezar Traykov255, Laura Morelli257,258, Luis Ignacio Brusco 240,241, Malik Rainer ${ }^{253}$, Mari Takalo ${ }^{175}$, Maria Bjerke ${ }^{196,270}$, Maria Del Zompo ${ }^{47,271}$, Maria Serpente ${ }^{80}$, Mariana Sanchez Abalos (10) ${ }^{272}$, Mario Rios ${ }^{229}$, Markku Peltonen ${ }^{142}$, Martin J. Herrman ${ }^{153}$, Mary H. Kosmidis ${ }^{169}$, Matias Kohler ${ }^{250,256}$, Matias Rojo ${ }^{240}$, Matthew Jones ${ }^{231,273}$, Michela Orsini ${ }^{236}$, Nancy Medel 268 , Natividad Olivar240, Nick C. Fox 266,274, Nicola Salvadori ${ }^{160}$, Nigel M. Hooper ${ }^{232}$, Pablo Galeano $244,257,258$, Patricia Solis ${ }^{268}$, Patrizia Bastiani ${ }^{171}$, Patrizia Mecocci ${ }^{171}$, Peter Passmore ${ }^{238}$, Reinhard Heun ${ }^{261}$, Riitta Antikainen275,276,277, Robert Olaso ${ }^{233}$, Robert Perneczky 167,278,279,280, Sandra Germani240, Sara López-García2,39, Seth Love 182,281, Shima Mehrabian ${ }^{255}$, Silvia Bagnoli66, Silvia Kochen ${ }^{268}$, Simona Andreoni ${ }^{100}$, Stefan Teipel 282,283 , Stephen Todd ${ }^{238}$, Stuart Pickering-Brown ${ }^{254}$, Teemu Natunen ${ }^{175}$, Thomas Tegos ${ }^{163}$, Tiina Laatikainen ${ }^{172,284,285}$, Timo Strandberg275,286, Tuomo M. Polvikoski189,190, Vaclav Matoska ${ }^{287}$, Valentina Ciullo ${ }^{120}$, Valeria Cores $244,245,252$, Vincenzo Solfrizzi ${ }^{239}$, Viviana Lisetti ${ }^{160}$ \& Zulma Sevillano 268 
${ }^{227}$ University of Oxford (OPTIMA), Oxford, UK. ${ }^{228}$ OPTIMA, Department of Pharmacology, University of Oxford, Oxford, UK. ${ }^{229}$ Dirección de Atención de Adultos Mayores del Min., Salud Desarrollo Social y Deportes de la Pcia. de Mendoza, Mendoza, Argentina. ${ }^{230}$ Geriatrics Unit Fondazione Policlinico A. Gemelli IRCCS, Rome, Italy. ${ }^{231}$ Cerebral Function Unit, Greater Manchester Neurosciences Centre, Salford Royal Hospital, Salford, UK. ${ }^{232}$ Division of Neuroscience and Experimental Psychology, School of Biological Sciences, Faculty of Biology, Medicine and Health, University of Manchester, Manchester, UK. ${ }^{233}$ Centre National de Recherche en Génomique Humaine (CNRGH), Institut de Biologie François Jacob, CEA, Université Paris-Saclay, F-91057 Evry, France. ${ }^{234}$ Department of Neurology, Kuopio University Hospital, Kuopio, Finland. ${ }^{235}$ Insitute of Clinical Medicine-Neurology, University of Eastenr Finland, Kuopio, Finland. ${ }^{236}$ Institute of Neurology, Catholic University of Sacred Heart, Fondazione Policlinico Universitario A. Gemelli IRCCS, Rome, Italy. ${ }^{237}$ Research Laboratory, Complex Structure of Geriatrics, San Giovanni Rotondo, Fondazione IRCCS Casa Sollievo della Sofferenza, Foggia, Italy. ${ }^{238}$ Centre for Public Health, School of Medicine, Queen's University Belfast, Belfast, UK. ${ }^{239}$ Clinica Medica "Frugoni" and Geriatric Medicine-Memory Unit, University of Bari Aldo Moro, Bari, Italy. ${ }^{240}$ CENECON-FMED-UBA, Buenos Aires, Argentina. ${ }^{241}$ ENERI, Vienna, Austria. ${ }^{242} \mathrm{Htal}$ Santojani, Buenos Aires, Argentina. ${ }^{243}$ Servicio de Neurología, Hospital de Cabueñes, Gijón, Spain. ${ }^{244}$ UBA, Buenos Aires, Argentina. ${ }^{245} \mathrm{HIGA}$ Eva Perón, Billinghurst, Argentina. ${ }^{246}$ University of Oxford, Oxford, UK. ${ }^{247}$ Neurología Clinica, Madrid, Spain. ${ }^{248}$ German Center for Neurodegenerative Diseases (DZNE), Tübingen, Germany. ${ }^{249}$ Section for Dementia Research, Hertie Institute for Clinical Brain Research and Department of Psychiatry and Psychotherapy, University of Tübingen, Tübingen, Germany. ${ }^{250}$ Hospital Dr. Lucio Molas, Santa Rosa, Argentina. ${ }^{251}$ Fundacion Ayuda Enfermo Renal y Alta Complejidad (FERNAC), Santa Rosa, Argentina. ${ }^{252}$ CONICET Buenos Aires, Argentina. ${ }^{253}$ Institute for Stroke and Dementia Research (ISD), University Hospital, Ludwig-Maximilian University Munich, Munich, Germany. ${ }^{254}$ Faculty of Medical and Human Sciences, Institute of Brain, Behaviour and Mental Health, Manchester, UK. ${ }^{255} \mathrm{Clinic}$ of Neurology UH 'Alexandrovska', Medical University—Sofia, Sofia, Bulgaria. ${ }^{256}$ Fundacion Sinapsis, Santa Rosa, Argentina. ${ }^{257}$ Laboratory of Brain Aging and Neurodegeneration- FIL, Buenos Aires, Argentina. ${ }^{258}$ IIBBA (CONICET), Buenos Aires, Argentina. ${ }^{259}$ Salford Royal NHS Foundation Trust, Salford, England. ${ }^{260}$ Nuffield Department of Clinical Neurosciences, University of Oxford, Oxford, UK. ${ }^{261}$ Department of Psychiatry, University of Bonn, Bonn, Germany. ${ }^{262}$ Department of Radiology, University Hospital Bonn, Bonn, Germany. ${ }^{263}$ Department of Public Health, University of Helsinki, Helsinki, Finland. ${ }^{264}$ National School of Public Health, Madrid, Spain. ${ }^{265}$ South Ostrobothnia Central Hospital, Seinäjoki, Finland. ${ }^{266}$ Dementia Research Centre, Department of Neurodegenerative Disease, UCL Queen Square Institute of Neurology, University College London, London, UK. ${ }^{267}$ Department of Neuropsychiatry, Charité, Berlin, Germany. ${ }^{268}$ ENYS (Estudio en Neurociencias y Sistemas Complejos) CONICET-Hospital El Cruce 'Nestor Kirchner'-UNAJ, Buenos Aires, Argentina. ${ }^{269}$ Molecular Medicine Center, Department of Medical chemistry and biochemistry, Medical University of Sofia, Sofia, Bulgaria. ${ }^{270}$ Laboratory of Neurochemistry, UZ Brussel, Brussel, Belgium. ${ }^{271}$ Unit of Clinical Pharmacology, Teaching Hospital of Cagliari, AOUCA, Cagliari, Italy. ${ }^{272}$ Programa del Adulto Mayor-Min, Salud de la Pcia. de Jujuy, San Salvador de Jujuy, Argentina. ${ }^{273}$ Division of Neuroscience and Experimental Psychology, University of Manchester, Manchester, UK. ${ }^{274}$ UK Dementia Research Institute at UCL, UCL Queen Square Institute of Neurology, University College London, London, UK. ${ }^{275}$ Center for Life Course Health Research, University of Oulu, Oulu, Finland. ${ }^{276}$ Medical Research Center Oulu, Oulu University Hospital, Oulu, Finland. ${ }^{277}$ Oulu City Hospital, Oulu, Finland.

${ }^{278}$ German Center for Neurodegenerative Diseases (DZNE, Munich), Munich, Germany. ${ }^{279}$ Department of Psychiatry and Psychotherapy, University Hospital LMU Munich, Munich, Germany. ${ }^{280}$ Ageing Epidemiology Research Unit (AGE), School of Public Health, Imperial College London, London, UK. ${ }^{281}$ University of Bristol Institute of Clinical Neurosciences, School of Clinical Sciences, Frenchay Hospital, Bristol, UK. ${ }^{282}$ German Center for Neurodegenerative Diseases (DZNE), Rostock, Germany. ${ }^{283}$ Department of Psychosomatic Medicine, Rostock University Medical Center, Rostock, Germany. ${ }^{284}$ Finnish Institute for Health and Welfare, Helsinki, Finland. ${ }^{285}$ Joint Municipal Authority for North Karelia Social and Health Services (Siun Sote), Joensuu, Finland. ${ }^{286}$ Helsinki University Hospital, University of Helsinki, Helsinki, Finland. ${ }^{287}$ Department of Clinical Biochemistry, Hematology and Immunology, Na Homolce Hospital, Prague, Czech Republic.

\section{The GR@ACE study group}

C. Abdelnour 1,2, N. Aguilera1, E. Alarcon 1,31, M. Alegret ${ }^{1,2}$, A. Benaque ${ }^{1}$, M. Boada ${ }^{1,2}$, M. Buendia', P. Cañabate ${ }^{1,2}$, A. Carracedo ${ }^{18,218}$, A. Corbatón-Anchuelo6 ${ }^{6}$, I. de Rojas' ${ }^{1}$ S. Diego ${ }^{1}$, A. Espinosa ${ }^{1,2}$, A. Gailhajenet ${ }^{1}$,

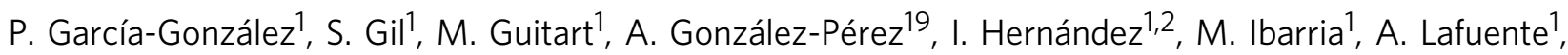
J. Macias ${ }^{23}$, O. Maroñas ${ }^{18}$, E. Martín', M. T. Martínez ${ }^{62}$, M. Marquié ${ }^{1}$ A. Mauleón', L. Montrreal', S. Moreno-Grau¹,2, M. Moreno¹, A. Orellana1, G. Ortega1,2, A. Pancho¹, E. Pelejá1, A. Pérez-Cordon¹, J. A. Pineda ${ }^{23}$, S. Preckler ${ }^{1}$, I. Quintela31, L. M. Real ${ }^{8,31}$, M. Rosende-Roca' ${ }^{1}$ A. Ruiz ${ }^{1,2}$, M. E. Sáez ${ }^{19}$, A. Sanabria ${ }^{1,2}$, M. Serrano-Rios ${ }^{61}$, O. Sotolongo-Grau', L. Tárraga ${ }^{1,2}$, S. Valero ${ }^{1,2} \&$ L. Vargas ${ }^{1}$

\section{DEGESCO consortium}

A. D. Adarmes-Gómez ${ }^{2,22}$, E. Alarcón-Martín1,31, M. D. Alonso ${ }^{288}$, I. Álvarez ${ }^{37,38}$, V. Álvarez ${ }^{26,138,}$ G. Amer-Ferrer ${ }^{289}$, M. Antequera ${ }^{13}$, C. Antúnez ${ }^{13}$, M. Baquero ${ }^{36}$, M. Bernal ${ }^{22}$, R. Blesa ${ }^{2,24}$, M. Boada ${ }^{1,2}$, D. Buiza-Rueda 2,22 , M. J. Bullido 2,215,216, J. A. Burguera ${ }^{36}$, M. Calero 2,21,40, F. Carrillo 2,22 , M. Carrión-Claro 2,22 ,

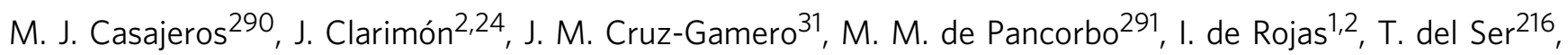
M. Diez-Fairen 37,38, R. Escuela2,22, L. Garrote-Espina2,22, J. Fortea 2,24, E. Franco-Macías ${ }^{22}$,

A. Frank-García 2,29,216, J. M. García-Alberca ${ }^{204}$, S. Garcia Madrona40, G. Garcia-Ribas ${ }^{40}$, P. Gómez-Garre2,22, I. Hernández ${ }^{1,2}$, S. Hevilla 204, S. Jesús 2,22, M. A. Labrador Espinosa2,22, C. Lage 2,39, A. Legaz ${ }^{13}$, A. Lleó2,24, A. Lopez de Munain32,33, S. López-García 2,39, D. Macias-García 2,22, S. Manzanares 289,292, M. Marín²2, J. Marín-Muñoz ${ }^{13}$, T. Marín ${ }^{204}$, M. Marquiée,2, A. Martín Montes²,29,215, B. Martínez ${ }^{13}$, C. Martínez ${ }^{26,243}$, 
V. Martínez ${ }^{13}$, P. Martínez-Lage Álvarez 293 , M. Medina 2,216, M. Mendioroz Iriarte ${ }^{294}$,

M. Menéndez-González 25,26, P. Mir ${ }^{2,22}$, J. L. Molinuevo ${ }^{295}$, L. Montrreal' ${ }^{1}$ A. Orellana', P. Pastor ${ }^{37,38,}$ J. Pérez Tur 2,212,213, T. Periñán-Tocino 2,22, R. Pineda-Sanchez 2,22, G. Piñol-Ripoll2,34,35, A. Rábano 2,21,46, D. Real de Asúa ${ }^{296}$, S. Rodrigo ${ }^{22}$, E. Rodríguez-Rodríguez 2,39, J. L. Royo ${ }^{31}$, A. Ruiz ${ }^{1,2}$, R. Sanchez del Valle Díaz ${ }^{14}$, P. Sánchez-Juan 2,39 , I. Sastre 2,215 , O. Sotolongo-Grau', S. Valero ${ }^{1,2}$, M. P. Vicente ${ }^{13}$, R. Vigo-Ortega ${ }^{2,22}$ \& L. Vivancos ${ }^{13}$

${ }^{288}$ Servei de Neurologia, Hospital Clínic Universitari de València, València, Spain. ${ }^{289}$ Department of Neurology, Hospital Universitario Son Espases, Palma, Spain. ${ }^{290}$ Hospital Universitario Ramón y Cajal, Madrid, Spain. ${ }^{291}$ BIOMICs, País Vasco, Centro de Investigación Lascaray, Universidad del País Vasco UPV/EHU, Leioa, Spain. ${ }^{292}$ Fundación para la Formación e Investigación Sanitarias de la Región de Murcia, El Palmar, Spain. ${ }^{293}$ Fundación CITA-alzheimer, Centro de Investigacio’n y Terapias Avanzadas, San Sebastián, Spain. ${ }^{294}$ Navarrabiomed, Pamplona, Spain.

${ }^{295}$ Barcelona beta Brain Research Center-Fundació Pasqual Maragall, Barcelona, Spain. ${ }^{296}$ Hospital Universitario La Princesa, Madrid, Spain.

\section{IGAP (ADGC, CHARGE, EADI, GERAD)}

C. Macleod ${ }^{297}$, C. McCracken ${ }^{298}$, Carol Brayne ${ }^{299}$, Catherine Bresner ${ }^{17}$, Detelina Grozeva ${ }^{17}$, Eftychia Bellou ${ }^{17}$, Ewen W. Sommerville ${ }^{17}$, F. Matthews ${ }^{300}$, Ganna Leonenko ${ }^{17}$, Georgina Menzies ${ }^{50}$, Gill Windle 297 , Janet Harwood ${ }^{17}$, Judith Phillips ${ }^{301}$, K. Bennett ${ }^{302}$, Lauren Luckuck ${ }^{17}$, Linda Clare ${ }^{303}$, Robert Woods ${ }^{304}$, Salha Saad ${ }^{17} \&$ Vanessa Burholt ${ }^{305}$

${ }^{297}$ School of Health Sciences, Bangor University, Bangor, UK. ${ }^{298}$ Institute of Psychology, Health and Society, University of Liverpool, Liverpool, England. ${ }^{299}$ School of Clinical Medicine, Cambridge Institute of Public Health, University of Cambridge, Cambridge, England. ${ }^{300}$ Faculty of Medicine, Institute of Health and Society, University of Newcastle, Callaghan, NSW, Australia. ${ }^{301}$ Dementia Studies, University of Stirling, Stirling, Scotland. ${ }^{302}$ School of Psychology, University of Liverpool, Liverpool, England. ${ }^{303}$ School of Psychology, Centre for Research in Ageing and Cognitive Health $(\mathrm{REACH})$, University of Exeter, Exeter, England. ${ }^{304}$ Dementia Services Development Centre, Bangor University, Wales, UK.

${ }^{305}$ Swansea University, School of Human Sciences, Centre for Innovative Ageing, Swansea, UK.

\section{PGC-ALZ consortia}

Iris E. Jansen ${ }^{3,9}$, Nancy L. Pedersen ${ }^{10}$, Arvid Rongve ${ }^{62}$, Chandra A. Reynolds (1) ${ }^{79}$, Dag Aarsland (10) 89,90, Geir Selbæk (1) 116,117,118, Ida K. Karlsson 10,133, Ingvild Saltvedt (13 ${ }^{135,136}$, Sara Hägg (1) ${ }^{10}$, Sigrid Botne Sando 201,202, Srdjan Djurovic (10) 206,207 , Ole A. Andreassen (10) 224,225 , Danielle Posthuma ${ }^{9}$ 